\title{
Smarte Kindheiten
}

Wenn junge Menschen in das Blickfeld städtischer Regierungsweisen rücken

\author{
Dana Ghafoor-Zadeh \\ Verena Schreiber
}

\begin{abstract}
Mit der Smart-City-Vision wird die Hoffnung verbunden, drängende ökologische, ökonomische und soziale Krisen in Städten zu überwinden und in eine ressourcenschonende und lebenswerte Stadt der Zukunft überzuleiten. Dabei fällt auf, dass Smart-City-Initiativen gezielt Kinder und junge Menschen adressieren. Anknüpfend an Ansätze der Gouvernementalitäts- und geographischen Kindheitsforschung gehen wir der Frage nach, wie die nahezu unhinterfragten Glaubenssätze einer von Innovationsoptimismus geprägten Stadt Vorstellungen von Kindheit verändern und ihre gesellschaftliche Inwertsetzung vorantreiben. Wir untersuchen erstens diskursanalytisch, wie Smart-City-Leuchtturmprojekte der EU in die Alltagswelten von Kindern investieren und diese als Experimentierfelder smarter Stadtentwicklung nutzen. Am Beispiel einer Fallstudie des Smart-City-Projektgebiets Wien-Simmering diskutieren wir zweitens, wie der smarte Stadtumbau auf das Alltagsleben von Kindern und Jugendlichen Einfluss nimmt und ihre Bedürfnisse be- und aufgreift. Unsere Ergebnisse zeigen, dass junge Menschen als smarte Bürger_innen in die Pflicht genommen werden, stadtentwicklungspolitische Zielsetzungen mitzutragen und entsprechende Handlungsweisen in ihren Alltag zu implementieren. Dies schafft neue Abhängigkeitsverhältnisse, die kritisch betrachtet werden müssen.
\end{abstract}

Ersteinreichung: 15. Januar 2021; Veröffentlichung online: 26. November 2021

An English abstract can be found at the end of the document.

\section{Einleitung}

Ein Freitagvormittagim Oktober 2019 in Wien: Zwischen den Prunkbauten der Innenstadt versammeln sich Hunderte junge Menschen. Sie halten Plakate hoch mit der Aufschrift „Actnow!“ oder „Wir schwänzen nicht Schule. Wir retten den Planeten! “. Im Namen von Fridays for Futuremachen Kinder undJ ugendlicheauf ihre Bedürfnisseaufmerksam und versuchen, diePolitik zu mehr Klimaschutz zu bewegen.

Ortswechsel - einige Kilometer südlich, im Wiener Bezirk Favoriten zur gleichen Zeit: Am Platz vor einer Kirche trudeln Schulklassen ein. „Hallo Kinder!“, schallt es aus Lautsprechern. Die Eröffnungsveranstaltung des Mobilitätsspiels „,Street-Points“ beginnt und der Moderator auf derimprovisierten Bühneam Kirchenportal motiviert die Schüler_innen zu Gymnastikübungen und Sprechchören. Immer wieder stimmt er sie ein: „Street-Points! Street-Points! Street-Points!“. 


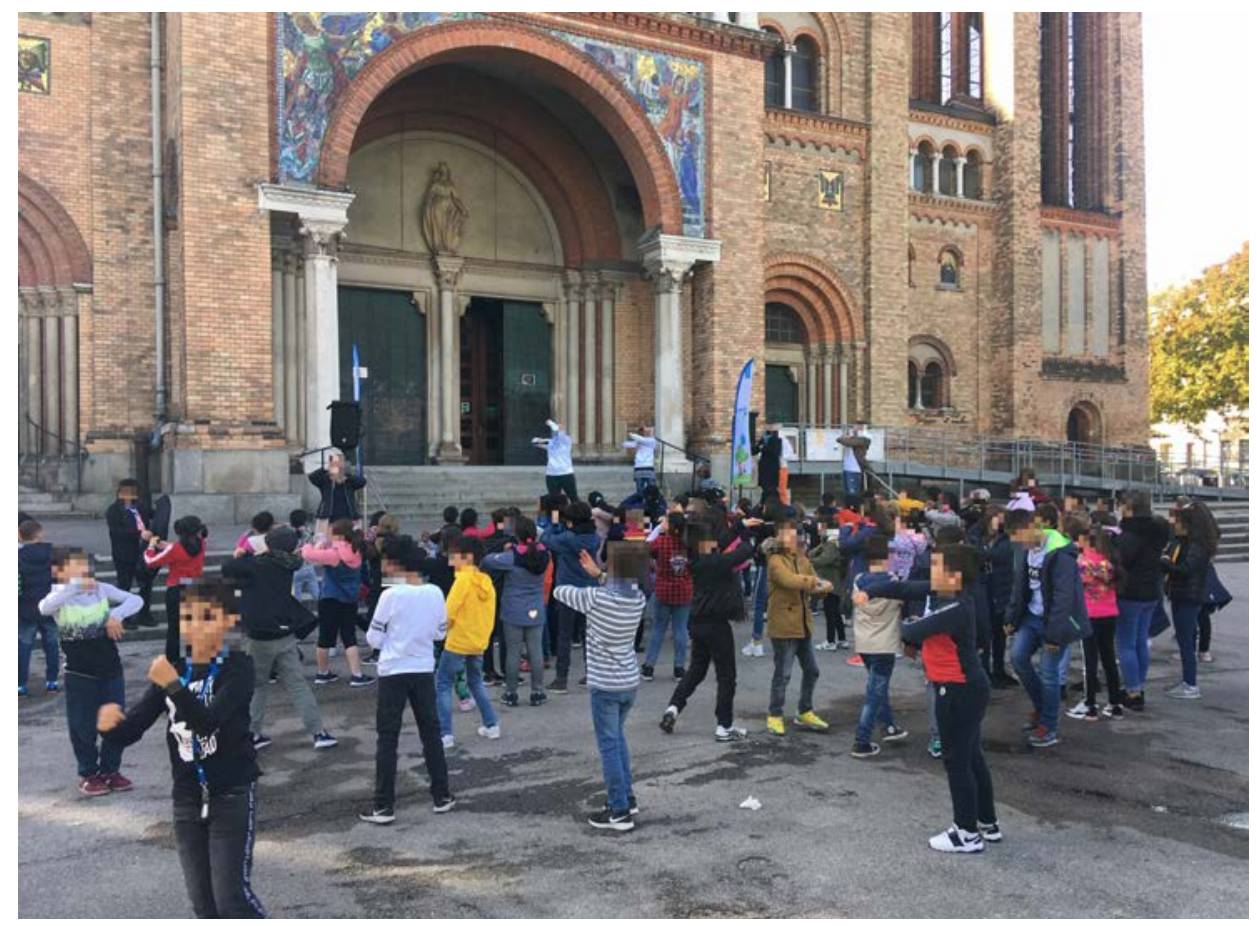

Abb. 1 Auftakt-

veranstaltung des

Mobilitätsspiels

"Street-Points" in

Wien-Favoriten

(Quelle: eigene

Aufnahme, 2019)

Während die Kinder gehorsam mitturnen (vgl. Abb. 1), erklärt dieWiener Fußgänger_innenbeauftragte, was die Kinder in den nächsten Wochen erwartet:

Fußgänger_innenbeauftragte: „Die Street-Points, das ist ein ganz ein cooles Spiel. Ihr habt alle schon so einen Chip, habe ich gesehen. “

Moderator: „Wo ist der Chip? Zeigt einmal her euren Chip.“

Fußgänger_innenbeauftragte: „Genau. Und mit diesem Chip könnt Ihr jetzt rund um die Schule an diesen Boxen, die Ihr immer wieder wo sehen werdet auf der Straße, Punkte sammeln. Und das Tolle ist: Eure Eltern, Geschwister, Großeltern können auch Punkte sammeln. Und wir wollen jetzt bis Ende November möglichst viel zu Fußgehen, damit wir allen zeigen, wie cool das ist, dass man in der Stadt viel zu Fuß geht. Es ist gesund, es macht uns schlau und ganz wichtig: Es ist auch ganz wichtig für unser Klima.“ (TB1)

2017 wurde im Rahmen der Smart-City-Initiative „Smarter Together“ im Wiener Bezirk Simmering das Mobilitätsspiel „Beat the Street“ entwickelt. Mit dem Folgeprojekt „Street-Points“ will man an den Erfolg von „Beat the Street" anknüpfen und auch in weiteren Bezirken fürmehr Bewegung zu Fuß, für mehr Gesundheit und Klimaschutz werben. Derselbe Tag, dieselbe Stadt, dieselbe Zielgruppe - doch unterschiedlicher könnten die Initiativen kaum sein: Auf der einen SeiteFridays for Futureals Bottom-up-Initiative, diemit zivilem Ungehorsam für einen Systemwandel eintritt; auf der anderen Seite die Smart-City-Initiative, diejunge Menschen top down zum Selbstwandel auffordert. Beide Male stehen Kinder und Jugendliche im Mittelpunkt. Beide Momentaufnahmen zeugen von ökologischen, ökonomischen und sozialen Krisen urbanen Lebens und ihren Bewältigungsversuchen.

Unser Beitrag setzt sich mit Kindheit in der Smart City auseinander. SmarteStadtvisionen haben in den letzten J ahren weltweit als Lösungen zur 
Bewältigunggegenwärtiger Herausforderungen an Bedeutung gewonnen. In den Smartness-Entwürfen nehmen digitaleTechnologien der Steuerungeine Schlüsselrolle ein: Mithilfe von „fließenden“ Informationen, Prognosen und Monitorings sollen städtische Prozesse effizienter organisiert, Bewegungsverläufe und Nutzungsverhalten der städtischen Bevölkerung optimiert sowie die Lebensqualität der Stadtbewohner_innen erhöht werden. Bei diesen Entwicklungen fällt auf, dass Smartness-Initiativen gezieltjunge Menschen adressieren, denen sie neue Zugangsmöglichkeiten zum öffentlichen Raum versprechen (van der Graaf 2020). Gleichzeitig aber nutzen sie ihre städtischen Lebensräume vermehrt als Experimentierfelder für smarte Transformationsprozesse. Dieser Beitrag setzt an dieser Beobachtung an und untersucht, wiejunge Menschen in Smart-City-Stadtentwicklungsprojekten in das Blickfeld städtischer Regierungsweisen rücken.

Anknüpfend an Ansätze der Gouvernementalitäts- und geographischen Kindheitsforschung gehen wir der Fragenach, wiedienahezu unhinterfragten Glaubenssätze einer von Technologie- und Innovationsoptimismus geprägten Stadt gängige Vorstellungen von Kindheit verändern und deren gesellschaftliche Inwertsetzung vorantreiben. Unter Rückgriff auf Planungs- und Dokumentationstexte zu Smart Cities sowie auf Expert_inneninterviews zeichnen wir nach, wie junge Menschen als „smart citizens“ (Shelton/ Lodato 2019; Cardullo/ Kitchin 2018) in die Pflicht genommen werden, stadtentwicklungspolitischeZielsetzungen mitzutragen, sich technologische Kompetenzen anzueignen sowieim AlltagentsprechendeHandlungsweisen und Optimierungsstrategien zu implementieren und weiterzuentwickeln. Anhand einer Fallstudie des Smart-City-Projektgebiets Wien-Simmering zeigen wir auf, wieder smarte Stadtumbau auf das Alltagsleben von Kindern und Jugendlichen Einfluss nimmt und ihre Bedürfnisse be- und aufgreift. Der Beitrag zieht abschließend eine kritische Bilanz der Beziehung zwischen Smart City und Kindheit und problematisiert neue Kindheitsvisionen und Abhängigkeitsverhältnisse, die aus dem Technikoptimismus smarter Stadtentwicklung erwachsen.

\section{Smart Cities im Spiegel der Gouvernementalitäts- und geographischen Kindheitsforschung}

Smart Cities folgen keinem einheitlichen Konzept. Hinter dem Begriff versammeln sich vielmehr unterschiedliche Transformationsprozesse und schillerndeVisionen für eine neue Stadtgesellschaft. Diese Vielschichtigkeit hat in den letzten J ahren eine ganze Reihe an Debatten angestoßen, die sich von konkret anwendungsbezogenen Fragen nach Infrastrukturen in den Bereichen Energie, Mobilität und Wohnungsbau über Governance und Sicherheit bis hin zu gesellschaftstheoretischer Kritik an einem naiven Fortschrittsoptimismus erstrecken. In einem Beitrag, der sich mit dem Verhältnis von Smart City und Kindheit befasst, wird nicht zuletzt der Begriff der Kindheit selbst bedeutsam, der in konzeptueller wie gelebter Weise mindestens so variantenreich ist wie Vorstellungen der Smart City selbst. Für unsere Auseinandersetzung werden daher zwei konzeptuelle Ansätze relevant: erstens Arbeiten, diesich aus einer gouvernementalen Perspektive mit smarten Steuerungsformen in urbanen Räumen auseinandersetzen und 
zweitens sozialwissenschaftliche Arbeiten zum Kind-Stadt-Verhältnis, die sich auf internationaler Ebene unter der Bezeichnung der Geographies of Childhood etablieren konnten, in Deutschland bislang aber nur am Rande rezipiert werden.

\subsection{Gouvernementale Steuerungsformen in der Smart City}

In der Stadtforschung haben sich in den letzten J ahren zahlreiche Studien mit der beschleunigten Digitalisierung und ihrem Einfluss auf Raumdarstellungen und -wahrnehmungen befasst. Dabei sind insbesondere Smart Cities ins Zentrum der Aufmerksamkeit gerückt. Mit dem dahinterliegenden Konzept einer intelligenten, digitalen Vernetzung von Infrastrukturen wird die Hoffnung verbunden, städtische Abläufe zu optimieren und hierdurch das Leben in den Städten in all seinen Facetten - Verkehr, Sicherheit, Ökologie, Partizipation etc. - zu verbessern (Kitchin 2014: 2; Greenfield 2013: 24; Hollands 2008: 307).

Vonseiten derkritischen Stadtforschungwurdedarauf hingewiesen, dasses der Debatteum Smart Cities an einer theoretischen Fundierungund Klärung zentraler Prämissen mangelt, und darauf hingewirkt, die ambivalenten gesellschaftlichen und sozialräumlichen Implikationen stärker in den Blick zu nehmen, die mit der Digitalisierung des Städtischen einhergehen (Rose 2020; Bauriedl/Strüver 2017). Die Smart City diene vor allem dazu, gesellschaftliche Krisen abzuwehren sowieneueVerantwortlichkeiten bei der Problembewältigung zu schaffen (Kropp 2018: 39f.; Gabrys 2014: 44). SmartCity-Konzepte und -Initiativen ordneten sich reibungslos in eine unternehmerische Stadtpolitik ein, die weniger auf die tatsächlichen Bedürfnisse von Stadtbewohner_innen abzielt, sondern sich primär der Entwicklung effizienter Technologien verpflichtet fühlt (Bauriedl/Strüver 2018: $18 \mathrm{f}$.). Kitchin (2014) sowie Shelton, Zook und Wiig (2015) verweisen hierbei auf die Hoffnung vieler Städte, durch Digitalisierungsprozesse Einsparungen und Innovationen vereinbaren zu können. In ähnlicher Weise argumentiert auch Hollands (2015), der in der Smart City vor allem eine Marketingstrategie zur Anwerbung finanzkräftiger Investor_innen vermutet. Drängende Probleme wie Armut und Ungleichheit dagegen würden durch diePriorisierungtechnischer Lösungen aus dem Blickgeraten (Söderström/ Paasche/Klauser 2014: 317). Ein weiteres Kritikfeld spannt sich um die Frage auf, inwiefern smarte Verwaltungs- und Infrastrukturen Asymmetrien beim Zugang zu städtischen Ressourcen und Beteiligungsprozessen abmildern oder aberverstärken (Bauriedl/Strüver 2018; Cardullo/ Kitchin 2018; Greenfield 2017; Datta 2015). Nicht zuletzt beruhe die Vision der Smart City auf europäischen Vorstellungen und Normen guten städtischen Lebens und fördere damit die „Universalisierung der europäischen Stadt“ (Bauriedl/ Strüver 2018: 14).

Für unseren Beitrag wird vor allem ein Diskussionsstrang innerhalb dieser Debatten relevant, der sich aus einer gouvernementalen Perspektive mit neuen Steuerungsformen in urbanen Räumen auseinandersetzt (Marquardt/ Schreiber 2021; Marquardt 2019; Rosol 2015; Füller/ Michel 2012; Pløger 2008). So prägen sich in smarten Transformationsprozessen deutlich gouvernementale Lenkungsformen durch, die auf die Schaffung 
einer sich selbst regulierenden, wettbewerbsfähigen Stadtgesellschaft zielen und unerwünschte Nebenfolgen einzudämmen versuchen (Lindner 2018; Gabrys 2014; Klauser/ Paasche/ Söderström 2014). Die Besonderheit gouvernementaler Steuerung liegt in ihren Kommunikationsformen und Wirkungsweisen: Diese basieren weniger auf der unmittelbaren Demonstration staatlicher Macht oder der Androhung von Strafen, sondern flechten vielmehr (digitale) Anreiz- und Kontrollsysteme in die Alltagspraktiken der Bevölkerung ein, um erwünschte Handlungsweisen zu begünstigen (Barry 2020).

Gouvernementale Regierungsformen fordern die Bevölkerung dazu auf, sich innovativen Entwicklungen anzuschließen, verstärkt Verantwortung für gesellschaftliche Problemlagen zu übernehmen sowie Kompetenzen zur Lösung dieser Probleme zu entwickeln (Lessenich 2012: 57). Individuen erfahren damit Veränderungen ihrer Handlungsweisen als selbstgewollt und nicht als Wirkung disziplinierender Maßnahmen (Lemke 2008: 13; Rosol 2015: 270). In diesem Sinne konnten zuletzt verhaltensökonomische Steuerungsansätze (Ranchordás 2019), die unter Einsatz von datengesteuerten „Nudges“ (dt.: Stupsern) Emotionen und Handlungen zu lenken versuchen (J akubowski 2018; Lindner 2018: 168), an Popularität in der Smart-City-Debatte gewinnen. Pykett (2012: 233) sieht hierin eine Form der „neuroliberalen “ und paternalistischen Steuerung, bei der Menschen im Diensteder gesellschaftlichen Fürsorgein derVeränderungihrer Verhaltensweisen angeleitet werden. Dabei geraten tiefgreifende Aspekte des individuellen Lebens ins Visier gouvernementaler Steuerung, da diese sich nicht mehr nur auf rationales Handeln, sondern vielmehr auch auf emotionaleund affektive Verhaltensweisen und höchst persönliche Impulse und Wünsche konzentrieren (Barry 2020: 104 f.).

Dieser Beitragfokussiertinsbesondereauf diegouvernementaleAnsprache von Kindern in smarten Stadtentwicklungskonzepten. Als sogenanntedigital natives gelten sieals zentraleAkteur_innen derWeiterentwicklungund Verbreitung digitaler Technologien und Steuerungsformen. Im empirischen Teil des Beitrags gehen wir daher der Fragenach, inwieweit Smart-City-Projekte insbesondere junge Menschen und ihre Räume adressieren und in die Pflicht nehmen, bereits ab dem Kindesalter eine „smartmentality“ (Vanolo 2014) auszubilden, stadtentwicklungspolitische Zielsetzungen mitzutragen und entsprechende Handlungsweisen in ihren Alltag zu implementieren.

\subsection{Urbane Kindheit}

Dass sich die Smart City gezielt an Kinder richtet, ist kein Zufall. Das relativ junge Konzept „Kindheit“ ist seit seiner Entstehung zur Projektionsfläche vielseitiger Utopien, Mythen und Visionen geworden und engmit Stadtentwicklungsprozessen verbunden. Kinder gelten als „Hoffnungsträger für all jene Aufgaben, dieder Generation der Erwachsenen zwar Sinn geben, hinter deren Visionen einer besseren Welt sieselbst aber zurückbleibt“ (Berg2004: 497). Angesichtszunehmender Krisen seit derJ ahrtausendwendegewinntKindheit für dieökonomisch, ökologisch und politisch sichere Zukunft der Gesellschaft zusätzlich an Bedeutung (Katz 2008: 6).

Historisch lässt sich dieEntstehungvon Kindheit als Vision einer besseren Zukunft bis ins 18. J ahrhundert zurückverfolgen. In seiner „Geschichte der 
Kindheit" zeigt Philippe Ariès (1976 [1960]), wie im Zuge der Entstehung der Nationalstaaten ein neues wirtschaftliches und erzieherisches Interesse an Kindern entstand. In der Wissenschaft wurde Kindheit nunmehr im Rahmen verhaltens- und entwicklungspsychologischer Forschung als eine eigene Lebensphase geistiger und körperlicher Entwicklung konzipiert und die biologische „Unfertigkeit“ von Kindern zum Anlass von Erziehungs-, Schutz- und Kontrollmaßnahmen genommen (Lee/ Motzkau 2011: 9 f.). Um eine Erziehung im Sinne des Staates zu gewährleisten, wurde es außerdem notwendig, neue Räume verdichteter Regulierungen und fürsorgerischer Aufsicht zu schaffen, in denen möglichst alle Kinder auf ihre spätere Aufgabe innerhalb der Gesellschaft vorbereitet werden konnten (Bühler-Niederberger/ Sünker 2009: 159). Die Stadt spielte in diesem Prozess stets eine zentrale Rolle; sie wirkte mit ihren Territorialisierungsstrategien und Institutionen maßgeblich daran mit, dass Kindheit als eine eigenständige Lebensphase überhaupt erst entstehen und in gewünschter Weise gestaltet werden konnte(Schreiber 2020, 2018; Zeiher 2009; Zinnecker 1990). Dieso entstandenen Modellvorstellungen einer „normalen “ und „guten“ Kindheit finden bis heute Anwendung und erheben globalen Geltungsanspruch (Singer 2019: 125; Prout 2005: 31 f.; Qvortrup 1993: 113).

Unter dem Einfluss der konstruktivistisch ausgerichteten New Social Studies of Childhood (Jenks 2004; Alanen 1997; James/Prout 1997; Qvortrup 1993) erweiterte sich ab den 1990er Jahren das forscherische Interesse an Kindern. So konnten einschlägige geographische, sozial-ökologische und historische Studien (Muchow/ Muchow 1998; Katz 1994; Ariès 1976 [1960]) zeigen, dass Kindheit keineswegs einem universalen Verständnis folgt oder sich aus natürlichen, biologischen Entwicklungen ableiten lässt, sondern dass sie kontextabhängig in je spezifischer Weise gelebt, begriffen und hergestellt wird (Schreiber 2018: 308; Prout 2005: 55). Als Konsequenz aus der Einsicht, dass Kindheit ein veränderbares Konstrukt ist und sich relational in Abgrenzung zu Vorstellungen von Erwachsensein formiert (Alanen 1997), fordern auch geographische Kindheitsforscher_innen eine stärkere Berücksichtigung der räumlichen Handlungsfähigkeit von Kindern (Kraftl 2020; Schreiber 2017; Aitken 2001; Valentine/ Holloway 2000; Katz 1994).

Dabei stehen nicht nur Kindheitsnarrative und die Dekonstruktion einer vermeintlich universalen Kindheit im Fokus. Vielmehr prägen auch empirische Studien zum Alltagsleben von Kindern, zu ihren Raumwahrnehmungen und -nutzungen das Forschungsfeld (Horton/Kraftl/Tucker 2012; Skelton/ Aitken 2019; Holloway/ Pimlott-Wilson 2011; Holt 2011). Vor allem der städtische Raum und dort angesiedelte Institutionen der Kindheit werden in den Blick genommen: Infolge von Stadtsanierung, Wohnungserneuerung und Massenmotorisierung der europäischen Städte Mitte des letzten J ahrhunderts und der damit einhergehenden Ängsteum diekindliche Sicherheit wurden Kinder zunehmend von der Straße und aus dem öffentlichen Raum verdrängt; ein erweiterter Bedarf an verhäuslichten Handlungsräumen wurde notwendig (Zeiher 2009: 117; Zinnecker 1990: 155). Während sich der Bewegungsradius vieler (männlicher) Erwachsener durch den Ausbau von Infrastrukturen stetig erweiterte, reduzierte sich dieser im Gegenzug für Kinder auf wenige, oft „verinselte“ Bereiche in Wohnortnähe 
(Tonucci/ Rissotto 2001: 411). Gleichzeitig entstanden pädagogische und kommerzielle Einrichtungen in den städtischen Quartieren, in denen sich Kinder fortan den Großteil ihres Tages aufhielten. Die Entwicklung hin zu einer Institutionalisierung des Alltagslebens von Kindern lenkt die Aufmerksamkeit der geographischen Kindheitsforschung bis heute auf dieFrage, wie eine selbstständige Raumaneignungin der Stadtfür Kinder noch möglich ist beziehungsweise wie sie gefördert werden kann (Kogler 2019; Zeiher 2018).

In unserem Beitrag schließen wir an internationale Studien im Feld der Geographies of Childhood an, die sich zum Ziel gesetzt haben, die Erfahrungen, Praktiken und Beziehungen junger und jüngster Menschen zu ihren Umgebungen partizipativ zu erschließen (Kraftl 2020; Schreiber 2020, 2018; Holloway 2014). Während in der Vergangenheit vonseiten der Kindheitsforschung insbesondere auf fehlende Zugangsmöglichkeiten von Kindern zum öffentlichen Raum hingewiesen wurde (Zeiher 2018: 29 f.), geben aktuelle Transformationsprozesse und Visionen der Smart City Anlass dazu, die Frage städtischer Raumwahrnehmung und -nutzung von Kindern neu zu diskutieren (Ghafoor-Zadeh 2021; van der Graaf 2020). So verweist Datta (2015: 50) beispielsweise darauf, dass sich junge Menschen in Indien von Smartness-Initiativen nicht nur eine erweiterte Beteiligung an der Stadtgesellschaft versprechen, sondern Smart Cities zu einem „part of the dreams and aspirations of ,success' of a young urban population" (ebd.) avanciert seien. Daran schließt die Frage an, wie Smart-City-Visionen und -Rationalitäten es schaffen (wollen), zum Leitbild einer ganzen Generation zu werden, und wie Kinder und junge Menschen Smart-City-Transformationen erleben.

\section{Kindheit in der Smart City: Programmatiken und Umsetzungsformen}

Auf Basis der dargelegten analytischen Zugänge der Gouvernementalitätsund Kindheitsforschung stellen wir im zweiten Teil des Beitrags Ergebnisse aus unserem Forschungsprojekt zu Kindheit in der Smart City vor. Den Gegenstand unserer Untersuchung bilden (1) Planungs- und Dokumentationsliteratur sowie Programmdarstellungen von EU-finanzierten SmartCity-Leuchtturmprojekten und (2) Material aus Expert_inneninterviews und Gruppendiskussionen sowie Stadtteilbegehungen und teilnehmenden Beobachtungen, das im Rahmen einer Fallstudie in Wien-Simmering im September und Oktober 2019 und August 2020 erhoben wurde.

$\mathrm{Zu}$ (1): Weltweit sind in den vergangenen J ahren zahlreiche SmartCity-Stadtentwicklungsprogramme mit dem Ziel aufgesetzt worden, fortschrittliche, sichere, wettbewerbsfähige und resiliente Städte zu schaffen und dadurch die Lebensqualität von Stadtbewohner_innen zu verbessern. Die Europäische Union hat dieIdee der Smart City vor allem durch dieAusschreibung umfangreicher Fördermittel in den verschiedenen Regionen Europas verankert. Im Rahmen des EU-Forschungs- und Innovationsprogramms „Horizon 2020“ (EuropäischeKommission o.J .) stiegen Städte wie Dresden oder Valencia zu Smart-City-Leuchtturmprojekten auf und schlossen sich zu einem europäischen Stadtnetzwerk zusammen. Als Teil der Smart-CityCommunity der EU sind dieseneuen Stadtkonsortien dazu aufgefordert, fortschrittliche Technologien in den Bereichen Energie, Wohnen und Mobilität 
zu testen und die Anwendung informationstechnologischer Innovationen voranzutreiben (TheSmart Cities Information System 2020). In diesem Kontext wurden in vielen europäischen Städten ausgewählte Stadtteilezu SmartCity-Laboren, in denen städtische Institutionen mit privatwirtschaftlichen Unternehmen sowiemit wissenschaftlichen Einrichtungen kooperieren und gemeinsam Visionen eines smarten Stadtlebens entwerfen, die dann direkt vor Ort in den Alltag der Bevölkerung integriert werden (Bauriedl 2018; Späth/Knieling 2018). Mit Abschluss der Projektphasen ist das Ziel dieser Städtedann, bislang weniger smarte Regionen zur Replikation erfolgreicher Maßnahmen zu motivieren.

Für unser Forschungsprojekt wurden Planungsliteratur sowie Programmdarstellungen von 17 Smart-City-Leuchtturmprojekten diskursanalytisch ausgewertet. Der Datenkorpus setzt sich zusammen aus: erstens den Horizon2020-Arbeitsprogrammen 2014-2015, 2016-2017und 2018-2020, zweitens den Aktionsfeldern des Smart Cities Marketplace der Europäischen Innovationspartnerschaft für intelligente Städte und Gemeinden (EIP-SCC) und drittens den Zielsetzungen und Ergebnisberichten der Leuchtturmprojekte sowie deren Newsbulletins, in denen über den jeweiligen Stand der SmartCity-Initiativen informiert wird. Im Fokus der Diskursanalysestehen folgende Fragen: Welchen Stellenwert haben junge und jüngste Stadtbewohner_innen in den Visionen und Plänen von Smart Cities? Wie werden sie als eine (homogene) Gruppe von „smart citizens “ konstituiert und wie werden ihre Praktiken und Bedürfnisse verhandelt? Inwiefern wird ihnen die Pflicht auferlegt, diedigitaleStadttransformation mitzutragen und voranzutreiben?

$\mathrm{Zu}$ (2): In einem zweiten Schritt wurden im Rahmen einer Fallstudie zum europäischen Smart-City-Leuchtturmprojekt „Smarter Together" in Wien-Simmering dieWirkungsweisen einer konkreten Smart-City-Initiative untersucht. Wien verfolgt seit 2014 einestadtweiteSmart-City-Strategieund hatim Rahmen der benannten EU-Investitionsprogramme bereits zahlreiche Maßnahmen zur intelligenten Quartiersentwicklung umgesetzt (Stadt Wien, Stadtentwicklungund Stadtplanung 2014). DieSmart-City-Rahmenstrategie wurde2019 noch einmal inhaltlich und zeitlich bis 2050 erweitert. Zusätzlich wurdeim J ahr 2020 eineeigene Kinder- und J ugendstrategieimplementiert, die „Maßnahmen aller Ressorts, Fachabteilungen und Unternehmen hinter der gemeinsamen Vision, Wien zur kinder- und jugendfreundlichsten Stadt zu machen ", bündelt und diesich an zahlreichen Stellen explizit auf dieSmartCity-Rahmenstrategie bezieht(StadtWien, J ugendabteilung 2020; vgl. auch UNICEF 2020; Brown etal. 2019). Nach dem Motto „Ein kinderfreundliches Wien ist ein klimafreundliches Wien und umgekehrt" (ehemalige Vizebürgermeisterin Hebein, zit. nach: Stadt Wien 2020), greifen in Wien folglich zwei aktuelle kommunale Planungsstrategien ineinander, welche die Stadt für unser Forschungsanliegen prädestinieren: So werden Kinder und junge Menschen gesondert adressiert und alltägliche Orte von Kindern, wie etwa Schulen, als Räumefür smarteVeränderungen der Stadtgesellschaft genutzt.

Das Zusammengreifen von smarten Stadttransformationen und einer an den Bedürfnissen von Kindern orientierten Stadtentwicklung wird im Wiener BezirkSimmeringbesonders deutlich. Simmeringliegtim Südosten von Wien, ist baulich von Stadterweiterungen der 1920er und 1950er J ahre geprägt und durch eine U-Bahn-Linie gut an die Innenstadt angebunden (Leban/ 
Leban 2001: 8). Der Stadtteil wird in den Medien als ein Ort multipler Konflikte und Problemlagen verhandelt, wo Wohnen zwar „,billig wiesonst nirgendwo in Wien “sei, wohin esjedoch kaum Menschen ziehe, „diefür eineAufwertung sorgen könnten“ (Kocina 2015). In den vergangenen J ahrzehnten wurden in Simmering immer wieder umfangreiche Stadtentwicklungsmaßnahmen umgesetzt, was den Bezirk auch in das Blickfeld der Smart-City-Stadtplanung rückte. Aufgrund des anhaltend hohen Sanierungsbedarfs wurde Simmering 2015 als Laborgebiet für den Umbau zu einem Smart-City-Leuchtturm-Ort im Rahmen des Projekts „Smarter Together - gemeinsam g'scheiter" ausgewählt und mit den Stadtteilen Lyon-Confluence und Neuaubing-Westkreuz/ Freiham (München) zu einem Konsortium für smarte Lösungen hinsichtlich einer nachhaltigen urbanen Entwicklung zusammengeführt (Smarter Together - gemeinsam g'scheiter 2020). Von 2016 bis 2019 wurden im Stadtteil vielfältige Maßnahmen implementiert, die auf Verbesserungen in den Bereichen Energie, Wohnen und Mobilität fokussieren.

Im Rahmen unserer Fallstudiewurden insgesamt 15 Expert_inneninterviewsmitAkteur_innen derWiener Smart-City-Rahmenstrategie, der SmartCity-Initiative „Smarter Together" sowie mit involvierten Institutionen im Bezirk Simmering geführt. Zudem nahmen wir an Veranstaltungen der Initiative teil, führten Interviews und Gruppendiskussionen mit Kindern und Jugendlichen im Alter zwischen 6 und 13 Jahren und initiierten einen Stadtteilrundgang mit jungen Menschen. Die Datenerhebung erfolgte im Herbst 2019 und im Sommer 2020 in zwei Horten, einer Schule und innerhalb der offenen Kinder- und J ugendarbeit in einem Stadtteilpark. Die Gespräche wurden mithilfe erzählgenerierender Stimuli wie selbst erstellten Stadtteilkarten, Fotografien des Stadtteils sowie Begriff-Charts unterstützt. Die Teilnahme der Kinder und J ugendlichen war freiwillig und erforderte das Einverständnis ihrer Erziehungsberechtigten. Ziel der Forschung mit Kindern war es, zu verstehen, wie Smart-City-Initiativen die gelebte Kindheit vor Ort beeinflussen, in welcher Weise Kinder in digitale Stadttransformationen eingebunden werden und wiesich die Realisierungen zu den Lebenswirklichkeiten und Bedürfnissen junger Menschen verhalten.

Die beiden Forschungszugänge der Diskursanalyse und der Fallstudie machen zwei spannungsreiche Problemfelder sichtbar, die wirim Folgenden ausführen: Wir diskutieren erstens, wie Smart-City-Leuchtturmprojekte der EU explizit in Alltagswelten von Kindern vordringen und wie sie Räume der Kindheit als Experimentierfelder für smarte Stadtentwicklung nutzen. Zweitenslegen wir dar, wieKinder als Heilsbringer_innen für smarteGesellschaftsvisionen berufen werden, welcheKonsequenzen darauserwachsen und welche Verantwortlichkeiten ihnen in der Smart-City-Stadtteilentwicklung auferlegt werden.

\subsection{Räume der Kindheit als Experimentierfelder smarter Stadttransformationen}

Zentrale Begründung der millionenschweren Smart-City-Investitionsprogramme der EU, so zeigt unsere diskursanalytischeAuswertung, ist eine anhaltende Urbanisierung, die mit einem steigenden Ressourcenverbrauch einhergehe (AP1: 81). Um die komplexen Herausforderungen urbaner 
Gesellschaften zu bewältigen, seien nachhaltige Strategien und der Einsatz neuer effizienter Technologien und Dienstleistungen in den Bereichen Energie, Mobilität und Kommunikation notwendig. Ziel des Aufbausvon Smart-CityNetzwerken sei es vor allem, neue Märkte zu etablieren, „buyers' groups“ (AP2: 175) für innovative intelligente Stadtlösungen zu schaffen und damit den Boden für Innovationen zu ebnen. Die EU erhofft sich von diesen Forderungen, die europäischeInnovations- und Technologiewirtschaft zu stärken, die wirtschaftlicheWettbewerbsfähigkeit zu erhöhen und europäischeStädte in den Rang eines „global role model and market leader“ zu überführen (ebd.). Smart Cities zielen auf einesmarteBewohner_innenschaft (Cardullo/ Kitchin 2018; Shelton/ Lodato 2019; Vanolo 2014), wollen „smarte Köpfe“ anziehen und für sie gesundheitlich, ökonomisch und gesellschaftlich von Vorteil sein (AP1: 88).

Obgleich die Smart-City-Leuchtturmprojekte grundsätzlich ähnliche Schwerpunkte setzen und klimafreundliche, ressourcenschonende Stadträume schaffen sowie wirtschaftlich erfolgreich sein wollen, fällt bei der Analyse ihrer Selbstdarstellungen eine Umkehrung der Legitimation eines smarten Stadtumbaus im Vergleich zu den EU-Papieren ins Auge. Während vonseiten derEUinsbesondere auf ökonomischeZieleverwiesen wird, rücken die Projektdarstellungen die Bewohner_innen der Städte und Stadtteile in den Vordergrund - sowohl als Profiteur_innen als auch als zentrale Akteur_innen des Transformationsprozesses: Für sie soll ein „vielfältiges Programm“ umgesetzt werden, das dieStadt „lebenswert“ (NL1) macht, den Bewohner_innen Kosten erspart und siezu Teilen einer „vibrant“(W1) sowie ökonomisch und ökologisch nachhaltigen und stimulierenden Gemeinschaft werden lässt (COR1). Ein Stadtentwicklungsprojekt sei zuallererst smart, „weil es Menschen verbindet“, und erst dann, weil es „die Wirtschaft einbezieht, das partnerschaftliche Zusammenwirken aller unterstreicht" und die „Standortattraktivität international fördert" (NL2).

In der Projektkommunikation vieler Städte sind es demzufolge dieStadtbewohner_innen selbst, die Vernetzung schaffen und neue Infrastrukturen nutzen sollen, um dieStadt in einen smarten Raum zu verwandeln. Im Mittelpunkt stehe nicht nur die Erneuerung von Infrastrukturen, sondern ein Wandel der Bewohner_innenschaft, ihrer gelebten Praktiken und Glaubenssätze: „Engage the citizens in creating a high quality living environment that inspires environmentally aware decisions and new patterns of behaviour" (W2). So investieren viele Smart-City-Projekte in umfassende Kommunikationsstrategien und Outreach-Aktivitäten, die sich konkret an Kinder und J ugendliche richten. Ihr Ziel ist es, junge Menschen mit der Smart-City-Programmatik vertrautzu machen und siein zentralen Bereichen der Smartifizierung weiterzubilden, sodass sie in ihrem künftigen Handeln die Kernanliegen des Smart-City-Leitbildes beherzigen und weitertragen. Kinder sollen sich im Rahmen verschiedener Aktivitäten smarte Kenntnisse und Kompetenzen aneignen und sich so selbst zu „critical citizens“ erziehen, ihr Umweltbewusstsein verbessern und ,the world around them using energy, water and gas" (W3) lieben und respektieren.

Eine besondere Wirksamkeit versprechen sich Smart-City-Leuchtturmprojektevon der expliziten Integration von Alltagsräumen und Institutionen junger Menschen in smarte Transformationsprozesse. Vor allem Schulen 
Abb. 2 PhotovoltaikTischplatte vor zwei Schulen in Simmering (Quelle: eigene Aufnahme, 2020)

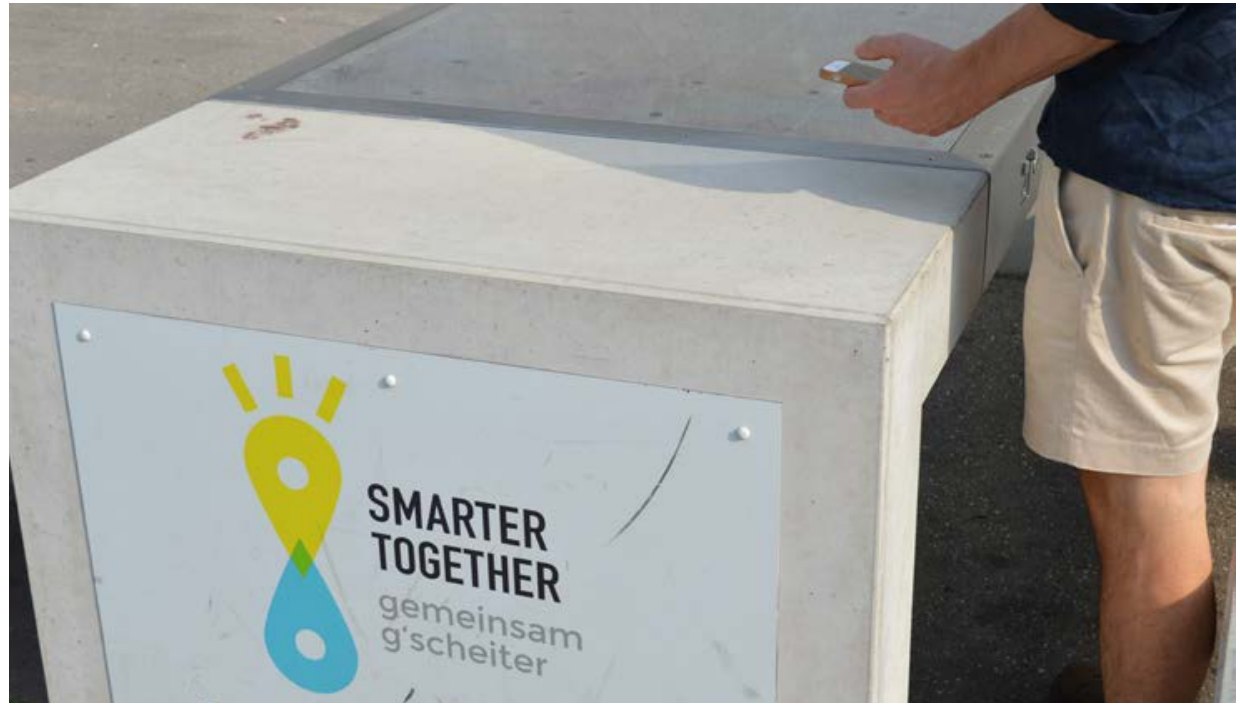

werden in einigen Projekten zu wichtigen Orten für smarteVeränderungen und zur Bühnefür dieinternationale Projektrepräsentation. Sanierungs- und Modernisierungsarbeiten sind ebenso wie „Verschönerungs“-Projekte, Feste und Spielaktionen Bestandteile von Smart-City-Leuchtturmprojekten und geben Raum für ein Doppelversprechen: Durch baulicheAufwertungen sollen erstens die Alltagsorte und Lernkontexte von Kindern verbessert werden. Zweitens sind „Kinder, die gerne in ihre Schule gehen, [...] einfach der beste Garant, dass sie ihre Chancen in Zukunft nutzen können und dass wir alle gemeinsam die Stadt weiterhin lebenswert gestalten“ (NL3). Von smarten Schulen erhoffen sich Stadtentwicklung und Politik eine Langzeitwirkung durch die Verankerung von Wissen, Fähigkeiten und positiven Visionen in den Curricula und Alltagserfahrungen der Heranwachsenden sowie durch die lokale Anbindung an zentrale Institutionen und wichtige Akteur_innen in den Bezirken. Schulen gelten als ideale Orte, an denen über Lehrpersonen und -inhalte, Rituale und Veranstaltungen neueThemen an Kinder und ihre Familien herangetragen werden können.

DieIntegration von Schulen bringt dasZiel dessmarten Stadtlebens bereits heute auf den Plan: „Everyone ranging from personnel to teachers and students areinvolved in all the energy management procedures and participate actively in all the decisions." (W3) Neben Kindern und Jugendlichen werden insbesondere auch Lehrpersonen durch ihre Mittlerfunktion zwischen Schule und lokaler Elternschaft und aufgrund ihrer anerkannten Autorität zu zentralen Agent_innen des Smart-City-Projekts:

„Nachdem wir quasi einen Wettbewerb hatten, die beste Schule bekommt die Turngeräte, konnten die Lehrer das - die wurden sehr intensiv vorher vorbereitet, ja - quasi vermitteln: Wenn die Kinder brav sind und viel spazieren, dann kriegen sie ein Turngerät." (IN1)

Lehrkräfte sind aufgefordert, Kinder zur Nutzung von smarten Infrastrukturen zu motivieren und damit abstrakte Visionen in neuen Gewohnheiten und Räumen konkret werden zu lassen. Smarte Gadgets sollen junge Menschen anregen, „umweltfreundliche Nachhaltigkeit bis in den Alltag“ zu leben (NL4). In diesem Sinne dienen im Rahmen der Smart-City-Projekte installierteSolar-Bänkeund Photovoltaik-Tischplatten (vgl. Abb. 2) auf dem Schulgelände nicht nur zum Aufladen von Smartphones. Sie sollen vielmehr 


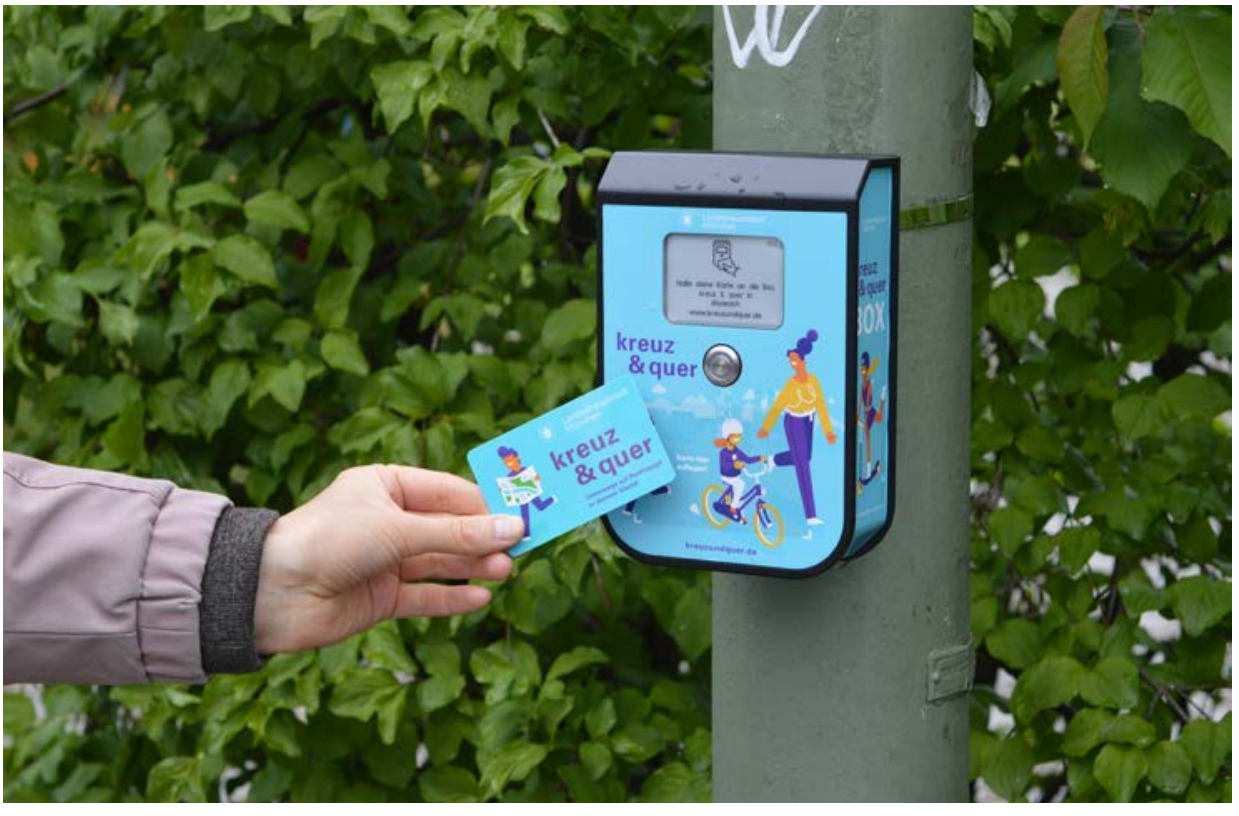

„dauerhaft an die Werte und Vision einer smarten und inklusiven Stadt mit Zukunftschancen erinnern“ (NL5) und werden damit für alle Bewohner_ innen zu einem „Denkmal“ für nachhaltige und innovative Stadtentwicklung.

Darüber hinaus initiieren Smart-City-Projekte eine ganze Reihe an Aktionen jenseits des engeren Schulumfelds, die sich gezielt an Kinder richten: Gemeinsam mit Wissenschaftsvereinen und Bildungseinrichtungen werden etwa Workshops veranstaltet, diejungen Menschen naturwissenschaftliches, ökologisches und informationstechnologisches Wissen näherbringen sollen. Auch eher spielerisch ausgelegteInitiativen wieder „Lighting Route“-Run für Schüler_innen im Rahmen eines Festakts für smarte Straßenlampen (NL6) oderdas Bemalen von Blumentöpfen fürdieBegrünungderStadt(NL7) bringen die Smartness-Mentalität in den Lebensraum von Kindern und involvieren diese gleichzeitig als tatkräftige Akteur_innen in die Projekte. Wettbewerbe wie das Energie-Quiz am „SIMmobil“ (NL8) oder ein Bus-Design-Wettbewerb (NL9) setzen weitereAnreize, zu partizipieren. Spielerischegouvernementale Erziehungsmethoden finden sich auch in ,interaktiven Geh- und Radlspielen“ (W4) wie dem eingangs erwähnten (vgl. Abb. 3).

Solche Spielelocken mit Gewinnchancen, während sie das Ziel verfolgen, eineÄnderung des Mobilitätsverhaltens von Kindern und ihren Familien herbeizuführen. Zunächst kurz- und dann langfristig sollen dadurch der Stadtraum ökologisch entlastet und Stadtbewohner_innen zu mehr Bewegungund einem gesunden Lebensstil angeregt werden. SpielerischeTechniken dienen dabei als subtile, aber wirkungsvolle Regierungsweisen und verwischen die Grenzen zwischen Freiwilligkeit und Verpflichtung (Vanolo 2018). Nach dem Motto „Introducing a more sustainable way of life through gaming“ (NL9) werden prominente Krisendiskurse zu Umwelt und Gesundheit durch Spiellogiken nicht nur forciert, sondern auch handhabbar gemacht. Stadtbewohner_innen sollen sich so auf niederschwellige Weise mit städtischen Problemen beschäftigen und die vorgetragenen Lösungsstrategien implementieren. Kinder und J ugendlicheerhalten beispielsweise Fahrräder „to promote modal shift, as part of a competition among schools to promote environmentally responsible behaviours" (NL10). E-Bikes mit Kindersitzen sollen es Familien ermöglichen, nachhaltige Mobilitätsformen zu nutzen
Abb. 3 Auch in Wiens Smart-City-Partnerstadt München wurde ein Mobilitätsspiel mit dem Titel „kreuz \& quer" durchgeführt. (Quelle: eigene Aufnahme, 2019) 
(W5). Paradoxerweise sind in Smart-City-Initiativen insbesondere solche Strategien - etwa richtige Müllentsorgungund mehr Bewegung- prominent, dieim Rahmen der Umweltbildungim Schulkontext schon seit J ahrzehnten behandelt werden. WieRäume der Kindheit als Experimentierfelder smarter Stadttransformationen genutzt werden, folgt demnach weniger innovativen Ideen, sondern zeigt sich als Fortsetzung und räumliche und zeitliche Ausweitung von Maßnahmen mit dem Ziel, Stadtbewohner_innen dazu zu bewegen, so früh und umfassend wie möglich etablierte Diskurse um „gute Lebensweisen" zu inkorporieren.

\subsection{Kinder als „Heilsbringer_innen“}

In den Programmen der Smart-City-Leuchtturmprojekte der EU wird zweitens das für neoliberale Gesellschaften typische Phänomen der zunehmenden Inwertsetzung von Kindheit (Katz 2008) sichtbar. Angesichts allgegenwärtiger Krisen werden junge Menschen als Potenzial für Sicherheit, Wachstum und Fortschritt stilisiert. Gesellschaften investieren in Kinder in der Hoffnung, ,that they actually will ,save the world“ or at least save us from ourselves and the consequences of our actions or inactions" (ebd.: 12). Dabei werden hohe und teils widersprüchliche Erwartungen an Kinder gestellt: Sie sollen einerseits in ihrem Kind-Sein als authentische, schnell lernende, neugierige, unvoreingenommene, optimistische und tatkräftige Akteur_ innen bestärkt werden. Andererseits sollen sie künftig als „erfolgreiche“ produktive Erwachsenezum wirtschaftlichen Erfolg beitragen, anstatt Sozialsysteme zu belasten (Redmond 2010: 479).

In der Charakterisierung von Smart-City-Leuchtturmstädten als lernende Städte (NL11) mit „Bildungsgrätzln“[1] und „student cities“ werden junge Menschen zu zentralen Agent_innen der Steigerungstädtischer Lebensqualität. J ugendlichkeit, Dynamik und neue Ideen scheinen Attribute zu sein, die sich in smarten Stadtteilen produktiv bündeln lassen: „This means the historic university town is always full of youth, life and new ideas which are turned into high-tech start-ups and companies." (W2) Die Art und Weise, in der junge Menschen zu Repräsentant_innen für eine aufstrebende Stadt der Zukunft werden, gleicht einer Dynamik, die Katz (2008) mit Blick auf neoliberale Gesellschaften als „childhood as ornament" beschrieben hat: „Ornament here refers to the museumification of the world, wherein there is a flattening of historical and geographical specificity that enables the projection of essences onto the object." (Ebd.: 14) Kinder werden damit zu einer homogenen Gruppe zusammengefasst, deren vermeintlich spezifische Eigenschaften es zu erhalten gelte. Die essenzialistische Vorstellung von Kindern als gesellschaftlichen Heilsbringer_innen - mystisch verklärt als halb übermenschliche, naturverbundene Wesen, die die Potenziale einer langen Lebenszeit und einer besonderen Verbindung zur natürlichen Welt haben (Taylor 2011) - ist grundlegender Teil dieser „Musealisierung“(ebd.). Wie sich in aktuellen Stadtentwicklungsprojekten zeigt, gilt einerseits die J ugendlichkeit selbst als wichtiges Attribut smarter Stadtvisionen. Gleichzeitig schaffen Smart-City-Initiativen vielfältige Formate für junge Menschen, bei denen neue Infrastrukturen nicht nur konsumiert werden, sondern sich Kinder selbst in die Gestaltung der Zukunftsszenarien ihrer Stadt einbringen 


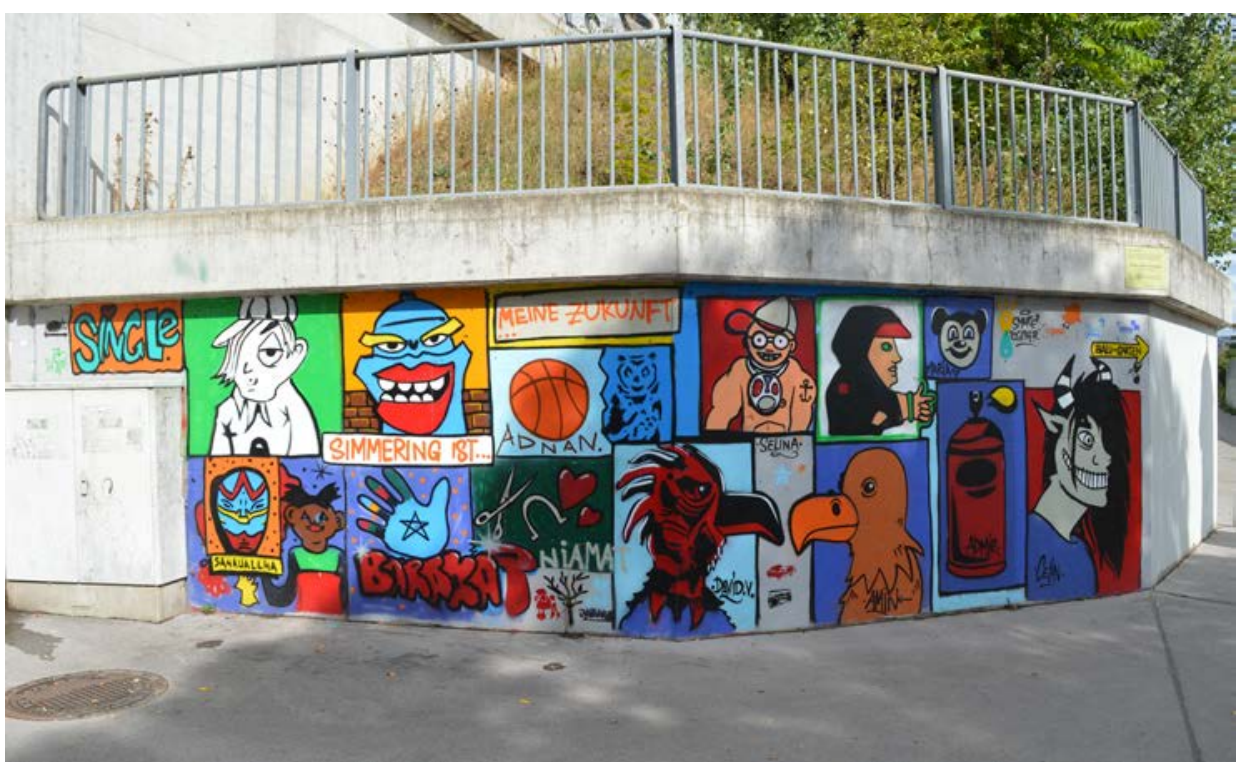

Abb. 4 Graffiti „Meine

Zukunft in Simmering"

(Quelle: eigene

Aufnahme, 2019)

sollen: „Thestudentsshould describe what thistown could look likein about50 yearsfrom now. "(NL12) UmjungeStadtbewohner_innen als ,smart citizens“ mit Vorbildfunktion sichtbar zu machen, wurden Kinder in einem Projekt beispielsweise dazu motiviert, Zukunftsvisionen für ihren Bezirk in Form eines Graffitis an die Wand einer S-Bahn-Station zu sprühen (vgl. Abb. 4).

Eine Strategie neoliberaler Gesellschaften im Umgang mit drohenden Krisen sei es, so Katz (2008: 14 f.), Kinder anzuregen, präventiv gegen ungewollte Zukunftsszenarien vorzugehen. Die Hoffnung, negative Entwicklungen abzuwenden, wird in Smart-City-Leuchtturmprojekten der EU besonders sichtbar und Kindern und Jugendlichen buchstäblich übergestülpt. J unge Menschen werden aufgefordert, zu "Climate Heroes“ im Stadtteil zu werden (NL13) und zusammen mit anderen „super Kids und Jugendliche[n], super Papis, super Mamis, super Omis und super Opis [...] und nicht zu vergessen: [den] super Lehrer_ innen“ aktiv eine „unglaublich nette Erfolgsgeschichte“ zu schreiben (NL5). Anstelledes Superman-Logos prangt der Schriftzug „I bin a smarter Simmeringer"/ „I bin a smarte Simmeringerin “ auf T-Shirts, die Schüler_innen bei der Eröffnung der im Rahmen einer Smart-City-Initiative sanierten und teilweiseneu errichteten Schulgebäude erhielten (vgl. Abb. 5).

Die politische Rationalität für die Zukunftssicherung städtischer Gesellschaften ganz besonders bei jungen Menschen anzusetzen, basiert vor allem auf neuen Kommunikations- und Regierungstechniken. Wie zuvor dargestellt, geht es in der Vermittlung smarter Themen zum einen darum, ein Verständnis für die Funktionsweisen smarter Technologien zu schaffen. Zum anderen steht, the promotion of an innovative business model such as energy and economic savings in schools" (W3) auf der Agenda der Smart-City-Projekte. Kinder und Jugendliche sollen als „Türöffner_innen“ agieren, um vor allem Familien und Erwachsene für die Smart-City-Vision zu gewinnen, die sich sonst kaum für stadtgesellschaftliche oder europapolitische Belange interessierten oder denen die Kompetenzen fehlten, neue Konzepte und Maßnahmen zu realisieren:

„Wir haben auch sehr viel über die Kinder argumentiert, weil mit Smart City, das haben wir schnell gemerkt, das interessiert keinen Menschen, da glauben die Leute: Das ist irgendwas von der Industrie 
Abb. 5 T-Shirts für Schüler_innen: „I bin a smarter Simmeringer" / „I bin a smarte Simmeringerin" (Quelle: eigene Aufnahme, 2019)

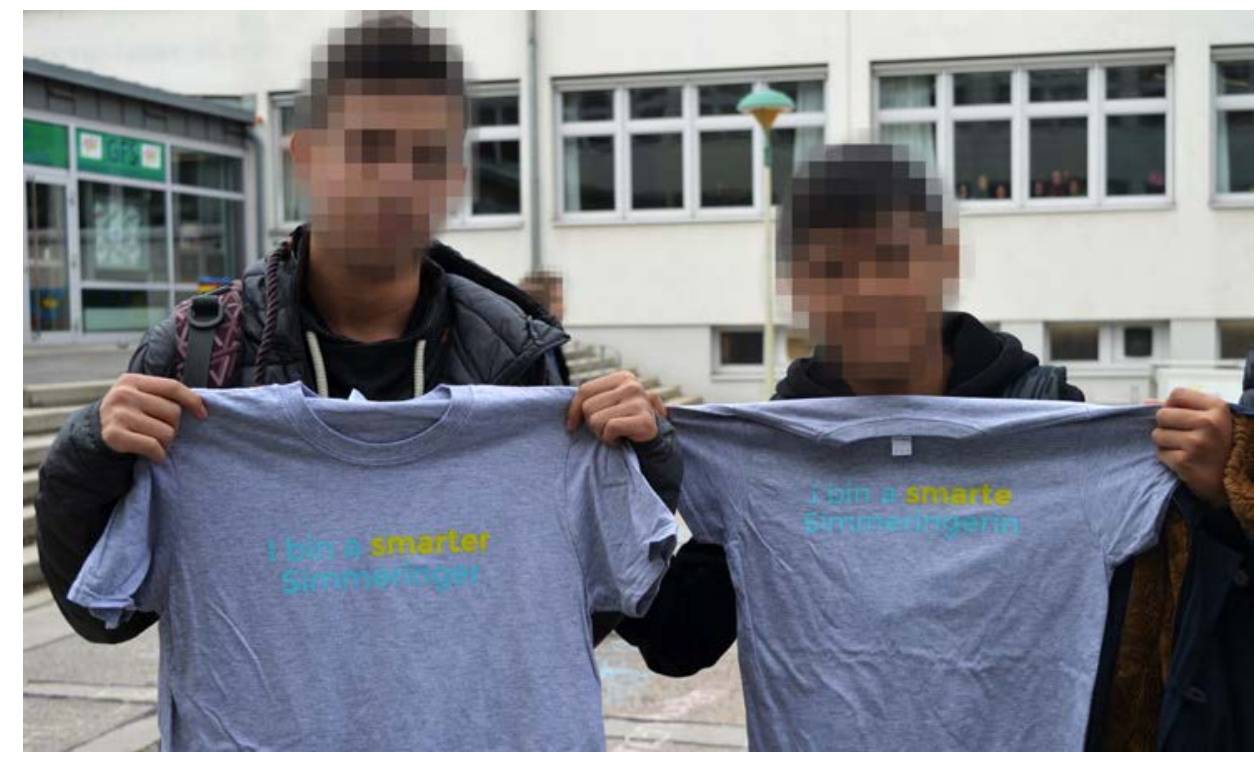

und von Europa und das ist so englisch. [...] Wir haben immer gesagt: ,WelcheStadt wollen wirfür unsere Kinder, Enkelkinder, Patenkinder, Nichten, Neffen, manche haben ja keine eigenen Kinder [...], welche Stadt wollen wir ihnen hinterlassen, ja? Und das ist eigentlich das beste Argument. [...] Und da haben sie dann keine Angst, dass das jetzt irgendwie konzerngesteuert ist oder von irgendwelchen Eliten [...] ausgedachte Konzepte sind, sondern da geht es wirklich im Kern um ihren Stadtteil. Und eben die meisten haben ja ingendwo Kinder oder Nichten, Neffen, die ihnen viel wert sind. Und das war eigentlich immer der icebreaker." (IN2)

Kinder werden hier erstens als „unvoreingenommener“ (IN2) und damit zugänglicher für die Smart-City-Initiativen dargestellt. Zweitens erscheinen Kinder als lokal verankerte Subjekte, denen eine höhere Affinität zum Stadtteil zugesprochen wird als Erwachsenen - und für den hätten sie auch einzutreten. Drittens wird Kindern eine besondere Vertrauens- und Glaubwürdigkeit attestiert, die sich durch ihre Position als Kind begründe. Diese könne dem mit der globalisierten und neoliberalisierten Stadtentwicklung einhergehenden Vertrauensverlust (Gerhard/Keller 2019: 301) entgegenwirken und dieLückezwischen Bürger_innen, Stadtentwicklungund Politik schließen:

„Wenn man ein bisschen großzz̈̈iger ist, dann helfen solche Projekte mit Kindern als Anstoß auch, um eine ganze Dynamik zu entwickeln. Auch mehr Glaubwürdigkeit zu haben und attraktiv zu sein. Wo dann alle möglichen sagen, ja, da möchte ich auch mitmachen." (IN1)

Smarte Regierungstechniken in EU-Leuchtturmprojekten agieren nicht im Modus von Geboten und Verboten, sondern setzen Impulse, sich als „enablers" zu verstehen. Damit dieseImpulseihreWirkungentfalten können, werden Dienste notwendig ,that support people in their own motivations to engage, express ownership, and change behaviour" (W6). Selle (2011: 11) konstatiert den unbändigen Drang von Stadtentwicklungspolitk nach mehr Beteiligungder Bewohner_innenschaft schon seitJ ahren: „Das Heischen um Aufmerksamkeit, die öffentlichkeitswirksame Inszenierung von Verfahren, 
die L'art-pour-l'art-Nutzung ,sozialer Medien' - alles das weist in diese Richtung.“ (ebd.) Im Projekt „Smarter Together“ in Wien-Simmering zeigt sich in besonderer Weise, dass sich die Aufmerksamkeit immer auf zwei Seiten richtet. Erstens soll Wien nach außen auf internationaler Bühne als smarteStadt attraktiv wirken, um dieeigeneWettbewerbsfähigkeitlangfristig zu erhöhen:

„Es gibt einen Wettbewerb um die besten Köpfe, global, ja. Es gibt auch einen Wettbewerb um Forschungseinrichtungen, es gibt einen Wettbewerb einfach um innovative Menschen. Auch hier das Signal rauszusenden: Wien ist so verfasst, dass es nicht nur ein guter Ort zum Leben ist, sondern dass es auch in 20, 30 Jahren noch ein guter Ort zum Leben ist.“(IN3)

Zweitens geht es jedoch auch darum, „nach innen “ zu agieren und die Stadtbevölkerung auf die Smart-City-Programme aufmerksam zu machen, damit diese auch wirksam werden können: „Ich meine, wie gesagt, das ist eine Strategie und die muss man auf den Boden bringen." (IN4) Denn es fehle - so kam es in den Interviews immer wieder zur Sprache - den Bewohner innen an Bereitschaft, sich mit den Zielen und Belangen der Smart-CityInitiativen auseinanderzusetzen: „Die haben ihre J obs. Die sind oft schlecht bezahlt, sehr prekär. Warum sollen diesich überhaupt dieseZeitund Energie nehmen, um darüber nachzudenken, was jetzt vorihrer Haustüreanders sein könnte, und mir diese Zeit zu schenken?“ (IN2) Gleichzeitig hätte die lokale Bevölkerung „mehr Kinder“ (IN2) und damit eine Grundvoraussetzung für notwendige Veränderungen, um „im Kopf, im Mindset etwas [zu] ändern“ (IN5). Kinder seien potenziell besser erreichbar und könnten mit Spiel, Spaß, Gewinnmöglichkeiten und Geschenken gewonnen werden, auch weil hier problemlos Einzelaspekte des „,integrativen“, „ganzheitlichen “ Smart-CityWandels herausgegriffen und spielerisch vorgestellt werden, während andere Aspekte unkommentiert im Hintergrund bleiben können: „Gerade so Themen wie Mobilität und Stadtplanung oder so, das ist oft so abstrakt. Das muss einfach Spaß machen [...], damit die Leute sich damit auseinandersetzen. Und da bietet halt die Digitalisierung sehr, sehr viele Möglichkeiten. “(IN2)

DieBegeisterungund Bereitwilligkeit vieler Menschen, Transformationen unhinterfragt anzunehmen und mitzugestalten, hat sich Barry (2020) zufolge vor allem im Zuge der Digitalisierung mithilfevon Apps verstärkt. Besonders im Kontext von Online-Nutzungen wirke die Prozesskette ,trigger - actionreward" (Liu/Li 2016, zit. nach Barry 2020: 14). DiesemacheNutzer_innen geradezu süchtignach neuen Gelegenheiten, um emotional angesprochen zu werden, sich (an-)erkannt zu fühlen, neue Handlungsweisen anzuwenden und dafür belohnt zu werden (ebd.: 14 f.). Unsere Untersuchung lässt vermuten, dass digitale Ökonomien derart dominant in den Alltagspraktiken vieler Stadtgemeinschaften angekommen sind, dass deren Logiken auch in Feldern wirksam werden, die gar nicht oder nur in geringem Maße auf digitalen Technologien basieren. In der Smart City mit ihren „accessible, easy and fun" Dienstleistungen (W7) wird diese Form der Ansprachesichtbar. Der Fokus auf affektive und emotionale Potenziale verspricht die Akzeptanz von Transformationsangeboten und dieBereitschaft, diese Veränderungen selbst 
aktiv mit umzusetzen, sodass die Beteiligten nach Beendigung von Projekten hungrignach neuen Transformationsmöglichkeiten zurückbleiben.

\section{Die Beziehung von Smart City und Kindheit: Eine kritische Bilanz}

In unserem Beitragkonnten wirnachzeichnen, dass Smart-City-Leuchtturmprojekte sowohl auf die Schaffung neuer Märkte für innovative Technologien als auch auf die Verbesserung von Lebensqualität für die Stadtbevölkerung zielen. Diese unterschiedlichen Prämissen stehen hier nicht im Widerspruch. Vielmehr werden sie in der Kommunikation mit verschiedenen Zielgruppen unterschiedlich gewichtet und verstärken sich gegenseitig. Kinder sind dabei explizitund implizitim Mittelpunkt. Siewerden zugleich als Leidtragendeunsmarter Städtesowieals Heilsbringer_innen für eineökologisch, ökonomisch und sozial sichere, wettbewerbsfähige und aufstrebendeZukunftsstadt adressiert. Eine „smart citizenship“, wiesiedie Smart City in glanzvollen Visionen, multimodalen Räumen, neuen Plattformen und Gadgets sowiemit innovativen Teilhabestrukturen anbietet, soll bewirken, dass Stadtbewohner_innen ihre Verhaltensweisen, Alltagsstrukturen und Gewohnheiten prüfen und mittel- und langfristig ändern. J unge Menschen erfahren dabei eine oft vermisste Aufmerksamkeit und einen in Zeiten omnipräsenter Krisendiskurse überraschenden Optimismus. Neue Organisationsstrukturen, veränderte Diskurs- und Beteiligungsformate, Investitionen in ressourcenschonende Technologien und teure Aufwertungsstrategien für Stadtgebieteversprechen, dass die smarte Stadt unverhoffte Handlungsmöglichkeiten schafft und Chancen auf gesellschaftliche Teilhabe neu verteilt.

„Cruel optimism“ nennt Berlant (2011) das siegessichere Streben nach etwas, das Glückverspricht, letztlich jedoch kontraproduktiv wirktund keine echten Chancen schafft. Auch die Smart City folgt einem geradlinigen und teils erbarmungslosen Technikoptimismus, mit dem Stadtbewohner_innen eine Aufwertung ihrer Lebensräume und eine neue Beachtung erfahren. Allerdings geschieht dies unter den Prämissen von Effizienzsteigerung und Optimierung von Massen und konzentriert sich auf nur wenige, für dielokale Bevölkerung zum Teil abseitige Themen und kaum auf deren tatsächliche Bedürfnisse. Eine direkte Auseinandersetzung der Stadt mit ihren Bewohner_innen und vice versa findet kaum statt. Damit fehlt es an echten Teilhabepotenzialen und an Investitionen an wichtigen Stellen. So stellt sich etwa die Frage, wo die Smart City Ressourcen und Raum für Experimente, alternative Lebensweisen, unproduktives Ausprobieren und Auffangnetze für Misserfolge zur Verfügung stellt.

Diese Beobachtung deckt sich mit den Untersuchungsergebnissen von Follmann, Leitheiser und Kretschmer (2021) zu Beteiligungsangeboten des Projekts „SmartCity Cologne“: Das enge Korsett der von Sparmaßnahmen und strikten Antragslogiken diktierten Stadtentwicklung für Innovationsförderung führt dazu, dass Zeit, Geld und Bereitschaft dafür fehlt, „eine Form der Bürger_innenbeteiligung [zu] bieten, bei der nicht nur unterschiedliche Interessen diskutiert werden, sondern auch emanzipatorisch-demokratisch [...] neue (digitale) Diskussionsräume zurZukunft der Stadt geschaffen werden“ (ebd.: 122). Stattdessen beschränken sich Städtein Smart-City-Konsortien auf 
eine zielführende „Alibi-Beteiligung“(ebd.: 134) der Bewohner_innen, um die erfolgreicheImplementierungohnegroßeWiderredesicherzustellen. Gleichzeitigbasiert das smarte Engagement auf einer instrumentalisierenden Haltung und defizitären Auffassung gegenüber der Bewohner_innenschaft. Trotz ihrer Bemühungen, mitKindern zusammenzuarbeiten, erfassen Smart-CityProjektekaum dieheterogenen Lebenswirklichkeiten oder dievielfältigen und weitreichenden Formen politischer und gesellschaftlicher Beteiligung von Kindern, ihre persönlichen Verwicklungen und sozialen Anteilnahmen (vgl. Mayall 2006; Skelton 2010; Kallio/Häkli 2013; Kallio 2018; Börner/Kraftl/ Giatti 2020). Vielmehrverfestigen sie generalisierendeZuschreibungen und zu kurz greifende Strategien. Kinder finden hier zwar eine neue Anerkennung. Allerdings basiert diese auf altersbezogener Differenzierung und macht sich den „Reichtum der Kinder“ (Blaisdell 2019) zu eigen. J unge Menschen erscheinen als kompetente Kraft der Stadt, von der Erwachsene lernen sollen. Gleichzeitig wird dabei erneut ein Unterschied in den generationalen Verhältnissen konstruiert. Ferner greift dievermeintliche Umkehr der generationalen Ordnung nur oberflächlich: Um kindergerecht zu erscheinen, werden Initiativen niedrigschwellig und unterkomplex geplant; Zusammenhänge und Prozesse werden stark vereinfacht dargestellt. Was gegenüber Erwachsenen möglicherweise als paternalistische, wenn nicht herabwürdigendeVorgehensweise beurteilt würde, gilt bei Kindern als alterssensibel und entwicklungsgerecht.

Unsere StudielässtdieSchlussfolgerungzu, dass dieBeziehungder Smart City zu jungen Menschen - so visionär und partizipativ sie daherkommen mag - auf bestehenden Zuschreibungen, fixen Vorstellungen, großen Visionen und hohen Erwartungen basiert. Nur an wenigen Stellen wird die Frage gestellt, um welche Kinder und um wessen Zukunft und Gegenwart es eigentlich gehen soll. Aufgrund der Dominanz von „adultist norms“ (Blaisdell 2019: 279) wird Partizipation von Kindern vor allem dann zum Thema, wenn sie zweckdienlich erscheint. Dagegen bleibt oft unbeachtet, in welch unterschiedlichen Formen Kinder ganz alltäglich am sozialen Leben teilnehmen (Mayall 2006). Kaum wird in Betracht gezogen, dass Kinder immer schon an der Gesellschaft partizipieren - allein schon, weil sie im Rahmen ihrer Schullaufbahn zum „Funktionieren komplexer Gesellschaften “ auf Basis von Wissen und Arbeitskraft mitwirken (vgl. ebd.: 15f.), vor allem aber, weil sie sehr wohl eigene Erfahrungen in und mit dieser Gesellschaft machen, eine Haltung zu Ereignissen und Diskursen einnehmen und sich in ihrem Alltag dazu verhalten (müssen) (Skelton 2010: $147 \mathrm{f}$.).

Dennoch fühlen sich viele junge Menschen von Smart-City-Maßnahmen angesprochen und beteiligen sich bereitwillig an den Initiativen, wie Gespräche mit Kindern und J ugendlichen gezeigt haben. Einige schätzten es, jenseits ihres üblichen Kontaktkreises Aufmerksamkeit zu erfahren, vor allem im Zuge der öffentlichen Berichterstattung. Manche nutzten zudem die Möglichkeit, im Rahmen der Initiative Dinge mit ihrer Familie oder Freunden zu tun, für die im Alltag sonst wenig Gelegenheit besteht - etwa gemeinsame Stadtspaziergänge im Rahmen von Mobilitätsspielen.

In unserer Studiezeigte sich auch, dass junge Menschen ebenso dringende und heterogene Bedürfnisse haben wie Erwachsene - und bei Weitem nicht so homogen sind, wiesiein der Smart-City-Programmatik konzipiert werden. 
Kinder gaben sich im Prozess der Datenerhebung genauso fragend, kritisch, kreativ und umweltbewusst wie desinteressiert, gleichgültig, antriebslos und unaufmerksam - und das oft unmittelbar nacheinander. So sprachen wir gerade über den hohen Stellenwert, den die Gruppe dem Naturschutz und dem richtigen Umgang mit Müll zurechnete, als eine Teilnehmerin ihr Bonbonpapier - nicht ungestraft vom Rest der Gruppe - auf eine Wiese warf (GD1). Ein Teilnehmer dachte viel über die harten Arbeitsbedingungen auf einer Großbaustelle im Stadtteil nach und kritisierte die geringe Vergütung auf dem Bau (GD2). Ein anderer Teilnehmer machtesich Gedanken über die Nutzung der öffentlichen Räume in Simmering: Die Parks im Bezirk seien zwar wichtige Treffpunkte für Jugendliche, allerdings viel zu gefährlich für jüngere Kinder. Alkohol- und Drogenkonsum, Konflikte mit anderen Personengruppen, Gewalt sowie eine hohe Polizeipräsenz mache die Stadtparks trotz Spielgeräten und Freiflächen zu eher kinder-unfreundlichen Orten (IG1).

Gerade junge und jüngste Menschen setzen sich in ihrem Alltag also mit gesellschaftlich drängenden Fragen auseinander. Dazu gehören Themen, die auch die Smart City forciert - wie etwa Umweltschutz, Klimawandel und Mobilitätsformen. Darüber hinaus fragen sienach Arbeitsbedingungen, thematisieren finanzielle Probleme, Freundschaften oder das Zusammenleben mit Tieren und Pflanzen. Auffällig ist dabei, dass auch diese Themen sehr wohl Bereichesind, diefür einezukunftsweisendeStadtentwicklung wichtig sind. Eine ernsthafte Einbeziehung junger Menschen wäre daher sowohl lohnend als auch notwendig.

Doch dieKomplexität des heterogenen und dynamischen Stadtlebens wird in lokalen Smart-City-Initiativen künstlich begrenzt und stark vereinfacht. Während spielerischeKommunikationsformen möglicherweise das Potenzial haben, Menschen freiwillig zur Beschäftigung mit stadtgesellschaftlichen Themen zu motivieren, ist fraglich, ob sie zu einer tiefergreifenden, kritischen Auseinandersetzungführen odervielmehr Gelegenheiten für dieErarbeitung alternativer Lösungswege verhindern. Unser Blick auf die SmartCity-Leuchtturmprojekte legt den Schluss nahe, dass Partizipation von Kindern besonders dann im Fokus steht, wenn greifbare und lösbare Herausforderungen benannt und mithilfeeinfacher Handlungsanweisungen angegangen werden können. Problemeohne Lösungsanleitungscheinen dagegen kaum Anlass fürpartizipativeInitiativen zu geben. Entgegen dem zunehmend komplexen Weltbild, das im öffentlichen Diskurs verhandelt wird, betont die Smart City gegenüberjungen Stadtbewohner_innen zu einfacheVorschläge, wie städtisches Zusammenleben aussehen kann. Paradoxerweise birgt ein solcher Ansatz von „dosierter" Teilhabe einerseits die Unterstellung, junge Menschen seien nicht in der Lage, sich mit komplexen Zusammenhängen auseinanderzusetzen, während er andererseits insbesondere Kinder zu vorbildlichen Akteur_innen der Smart City erziehen und ihnen in der Rolle von Multiplikator_innen und Botschafter_innen eine großeVerantwortung übertragen möchte. Kinder werden also nicht als „reflexive agents“ (Redmond 2010) angesehen, sondern vielmehr zu Ausführenden neuer Praktiken und Gewohnheiten für einen Mentalitätswandel im Sinne einer smarten Gesellschaft.

Der Beitrag hat sich dem spannungsreichen Verhältnis von Smart City und Kindheit gewidmet. Hier schließen sich zahlreiche weitere Fragen für 
geographische Forschung, etwa zu Nachhaltigkeitsdiskursen, Raumgestaltung und Umnutzungsformen an. Auch methodisch sehen wir wertvolle Anschlussstellen: etwa über repräsentative Methoden hinauszugehen und mithilfe kreativer Methoden insbesondere emotionale und affektive Dimensionen der Beziehung junger Menschen zu ihrem Stadtraum zu erschließen. Nicht zuletzt verweisen wir mit unserem Beitrag auf die Notwendigkeit, universale, vereinfachte und stigmatisierende Diskurse und Handlungsweisen gegenüber jungen Menschen aufzudecken und ihnen entgegenzuwirken.

Wir danken den Herausgeber_innen von su b \ u r ba n und den anonymen Gutachter_innen für ihre konstruktiven Kommentare zum Beitrag.

Die Publikation wurde mit Mitteln der DFG (SCHR 1329/2-1) und durch den Open Access-Publikationsfonds des Ministeriums für Wissenschaft, Forschung und Kunst Baden-Württemberg und der Pädagogischen Hochschule Freiburg gefördert.

\section{Endnoten}

[1] Der Begriff „Bildungsgrätzl“ bezeichnet in Österreich Kooperationen von Schulen und Kindergärten mit außerschulischen Einrichtungen im Stadtteil. Akteur_innen aus der Kinder-, J ugend- und Erwachsenenbildung sowie aus den Bereichen Sport, Kultur, Sozialarbeit und Gesundheit verfolgen dabei das Ziel, mithilfe vielfältiger Angebote den Stadtteil ganzheitlich zu einem innovativen, inklusiven und vernetzten Lernort zu machen.

\section{Autor_innen}

Dana Ghafoor-Zadeh forschtim Bereich Humangeographiemit dem Schwerpunkt Children's Geographies und Stadtgeographien.

dana.ghafoor@ph-freiburg.de

Verena Schreiber lehrt in den Bereichen Geographiedidaktik und Humangeographie und forscht zu aktuellen Stadtentwicklungsprozessen, Children's Geographies und feministischtransformativer Bildung.

verena.schreiber@ph-freiburg.de

\section{Literatur}

Aitken, Stuart C. (2001): Geographies of young people. The morally contested spaces of identity. London $u$. a.: Routledge.

Alanen, Leena (1997): Soziologie der Kindheit als Projekt. Perspektiven für die Forschung. In: Zeitschrift für Sozialisationsforschung und Erziehungssoziologie 17/ 2, 162-177.

Ariès, Philippe (1976 [1960]): Geschichte der Kindheit. München: Hanser.

Barry, Laurence (2020): The quantified self and the digital making of the subject. In: Matteo Stocchetti (Hg.), The digital age and its discontents. Critical reflections in education. London: Helsinki University Press, 95-110.

Bauriedl, Sybille (2018): Smart-City-Experimente. Normierungseffekte in Reallaboren. In: Sybille Bauriedl / Anke Strüver (Hg.), Smart City. Kritische Perspektiven auf die Digitalisierung in Städten. Bielefeld: transcript, 75-85. 
Bauriedl, Sybille/ Strüver, Anke(2017): SmarteStädte. DigitalisierteurbaneInfrastrukturen und ihre Subjekte als Themenfeld kritischer Stadtforschung. In: subl urban. zeitschrift für kritische stadtforschung 5/ 1-2, 87-104.

Bauriedl, Sybille / Strüver, Anke (2018): Raumproduktionen in der digitalisierten Stadt. In: Sybille Bauriedl / Anke Strüver (Hg.), Smart City. Kritische Perspektiven auf die Digitalisierung in Städten. Bielefeld: transcript, 11-30.

Berg, Christa (2004): Kind/Kindheit. In: Dietrich Benner / Jürgen Oelkers (Hg.), Historisches Wörterbuch der Pädagogik. Weinheim/ Basel: Beltz, 497-517.

Berlant, Lauren Gail (2011): Cruel optimism. Durham: Duke University Press.

Blaisdell, Caralyn (2019): Participatory work with young children. The trouble and transformation of age-based hierarchies. In: Children's Geographies 17/3, 278-290.

Börner, Susanne / Kraftl, Peter / Giatti, Leandro Luiz (2020): Blurring the „-ism“ in youth climate crisis activism. Everyday agency and practices of marginalized youth in the Brazilian urban periphery. In: Children's Geographies 19/3, 275-283

Brown, Caroline / Lannoy, Ariane de / McCracken, Deborah / Gill, Tim / Grant, Marcus / Wright, Hannah / Williams, Samuel (2019): Special issue. Child-friendly cities. In: Cities \& Health 3/1-2, 1-7.

Bühler-Niederberger, Doris / Sünker, Heinz (2009): Gesellschaftliche Organisation von Kindheit und Kindheitspolitik. In: Michael-Sebastian Honig (Hg.), Ordnungen der Kindheit. Problemstellungen und Perspektiven der Kindheitsforschung. Weinheim/ München: J uventa, 155-182.

Cardullo, Paolo / Kitchin, Rob (2018): Being a „citizen“ in the smart city. Up and down the scaffold of smart citizen participation in Dublin, Ireland. In: GeoJournal 84/4, 1-13.

Datta, Ayona (2015): A 100 smart cities, a 100 utopias. In: Dialogues in Human Geography $5 / 1,49-53$.

Europäische Kommission (o. J.): Horizon 2020. https://ec.europa.eu/programmes/ horizon2020/ en (letzter Zugriff am 11.1.2021).

Follmann, Alexander / Leitheiser, Stephen / Kretschmer, Holger (2021): Smart und/ oder partizipativ? Eine kritische Betrachtung der SmartCity Cologne. In: subl urban. zeitschrift für kritische stadtforschung 9/1-2, 115-139.

Füller, Henning / Michel, Boris (Hg.) (2012): Die Ordnung der Räume. Geographische Forschung im Anschluss an Michel Foucault. Münster: Westfälisches Dampfboot.

Gabrys, J ennifer (2014): Programming environments. Environmentality and citizen sensing in the smart city. In: Environment and Planning D: Society and Space 32/1, 30-48.

Gerhard, Ulrike / Keller, Judith (2019): „My home is my castle“ - über die Rolle von Vertrauen im Wohnungsbau. Ein Blick auf US-amerikanische Städte. In: Forum Wohnen und Stadtentwicklung 11/6, 300-304.

Ghafoor-Zadeh, Dana (2021): Kindheit. In: Tabea Bork-Hüffer / Henning Füller / Till Straube (Hg.), Handbuch Digitale Geographien: Welt - Wissen - Werkzeuge. Paderborn: utb Schöningh.

Greenfield, Adam (2013): Against the smart city. New York: Do Publications.

Greenfield, Adam (2017): Practices of the minimum viable utopia. In: Architectural Design 87/1, 16-25.

Hollands, Robert G. (2008): Will the real smart city please stand up? In: City 12/3, 303-320.

Hollands, Robert G. (2015): Critical interventions into the corporate smart city. In: Cambridge J ournal of Regions, Economy and Society 8/1, 61-77.

Holloway, Sarah L. (2014): Changing children's geographies. In: Children's Geographies 12/4, 377-392.

Holloway, Sarah L. / Pimlott-Wilson, Helena (2011): The politics of aspiration. Neo-liberal education policy, „low" parental aspirations, and primary school extended services in disadvantaged communities. In: Children's Geographies 9/ 1, 79-94.

Holt, Louise (Hg.) (2011): Geographies of children, youth and families. An international perspective. London: Routledge.

Horton, J ohn / Kraftl, Peter / Tucker, Faith J . (Hg.) (2012): Critical geographies of childhood and youth. Contemporary policy and practice. Bristol: Policy Press.

Jakubowski, Peter (2018): Nudging in der digitalen Stadt. Idee, Potenziale und kritische Reflexionen. Bonn: Bundesinstitut für Bau-, Stadt- und Raumforschung (BBSR) im Bundesamt für Bauwesen und Raumordnung (BBR).

James, Allison / Prout, Alan (Hg.) (1997): Constructing and reconstructing childhood. Contemporary issues in the sociological study of childhood. London: RoutledgeFalmer. 
J enks, Chris (2004): Many childhoods? Editorial. In: Childhood 11/1, 5-8.

Kallio, Kirsi Pauliina (2018): Not in the same world: Topological youths, topographical policies. In: Geographical Review 108/4, 566-591.

Kallio, Kirsi Pauliina / Häkli, Jouni (2013): Children and young people's politics in everyday life. In: Space and Polity 17/ 1, 1-16.

Katz, Cindi (1994): Textures of global change. Eroding ecologies of childhood in New York and Sudan. In: Childhood 2/1-2, 103-110.

Katz, Cindi (2008): Cultural Geographies lecture. Childhood as spectacle. Relays of anxiety and the reconfiguration of the child. In: cultural geographies 15/1, 5-17.

Kitchin, Rob (2014): The real-time city? Big data and smart urbanism. In: GeoJ ournal 79/1, $1-14$.

Klauser, Francisco / Paasche, Till / Söderström, Ola (2014): Michel Foucault and the smart city. Power dynamics inherent in contemporary governing through code. In: Environment and Planning D: Society and Space 32/5, 869-885.

Kocina, Erich (2015): Simmering: Der Prügelknabe der Wiener Bezirke. In: Die Presse, 18.9.2015. https:/ / www.diepresse.com/ 4824511/ simmering-der-prugelknabe-derwiener-bezirke (letzter Zugriff am 6.11.2020).

Kogler, Raphaela (2019): Räume für Kinder - Räume der Kinder. Typologien urbaner Kinderräume. In: Forum Wohnen und Stadtentwicklung 11/1, 11-14.

Kraftl, Peter (2020): After childhood. Re-thinking environment, materiality and media in children's lives. London: Routledge.

Kropp, Cordula (2018): Intelligente Städte. Rationalität, Einfluss und Legitimation von Algorithmen. In: Sybille Bauriedl / Anke Strüver (Hg.), Smart City. KritischePerspektiven auf die Digitalisierung in Städten. Bielefeld: transcript, 33-42.

Leban, Petra / Leban, Hannelore (2001): Simmering. Wiens 11. Bezirk. Erfurt: Sutton.

Lee, Nick/ Motzkau, J ohanna (2011): Navigating thebio-politics of childhood. In: Childhood 18/1, 7-19.

Lemke, Thomas (2008): Gouvernementalität und Biopolitik. Wiesbaden: VS Verlag für Sozialwissenschaften.

Lessenich, Stephan (2012): Der Sozialstaat als Erziehungsagentur. In: Aus Politik und Zeitgeschichte 62/49-50, 55-61.

Lindner, Peter (2018): Smart Cities - Smart Bodies? In: Sybille Bauriedl / Anke Strüver (Hg.), Smart City. Kritische Perspektiven auf die Digitalisierung in Städten. Bielefeld: transcript, $161-173$.

Marquardt, Nadine (2019): Oikopolitik. In: Geographische Zeitschrift 106/4, 212.

Marquardt, Nadine / Schreiber, Verena (2021): Wenn Raumproduktionen zu Regierungspraktiken werden. Michel Foucaults Angebote an die Geographie. In: Georg Glasze / Annika Mattissek (Hg.), Handbuch Diskurs und Raum (vollständig überarbeitete und erweiterte Ausgabe). Bielefeld: transcript, 195-206.

Mayall, Berry (2006): Values and assumptions underpinning policy for children and young people in England. In: Children's Geographies 4/ 1, 9-17.

Muchow, Martha / Muchow, Hans Heinrich (Hg.) (1998): Der Lebensraum des Großstadtkindes. Weinheim: Juventa.

Pløger, J ohn (2008): Foucault's dispositif and the city. In: Planning Theory 7/ 1, 51-70.

Prout, Alan (2005): The future of childhood. Towards theinterdisciplinary study of children. London: RoutledgeFalmer.

Pykett, J essica (2012): The new maternal state. The gendered politics of governing through behaviour change. In: Antipode 44/1, 217-238.

Qvortrup, Jens (1993): Die soziale Definition von Kindheit. In: Manfred Markefka / Bernhard Nauck (Hg.), Handbuch der Kindheitsforschung. Neuwied u. a.: Luchterhand, 109-124.

Ranchordás, Sofia (2019): Nudging citizens through technology in smart cities. In: International Review of Law, Computers \& Technology 34/3, 254-276.

Redmond, Gerry (2010): Children's agency and the welfare state. Policy priorities and contradictions in Australia and the UK. In: Childhood 17/4, 470-484.

Rose, Gillian (2020): Actually-existing sociality in a smart city. In: City 24/3-4, 512-529.

Rosol, Marit (2015): Governing cities through participation. A Foucauldian analysis of CityPlan Vancouver. In: Urban Geography 36/2, 256-276. 
Schreiber, Verena (2017): Forschen mit Kindern. In: Quasus. Qualitatives Methodenportal zur Qualitativen Sozial-, Unterrichts- und Schulforschung. https://quasus.ph-freiburg. de/ forschen-mit-kindern (letzter Zugriff am 15.1.2021).

Schreiber, Verena (2018): Kindheit. In: Bernd Belina / Matthias Naumann / Anke Strüver (Hg.), Handbuch Kritische Stadtgeographie. Münster: Westfälisches Dampfboot, 306-311.

Schreiber, Verena (2020): Geographien der Kindheit. Zur Relevanz des Räumlichen für die Kindheitsforschung. In: Sabine Bollig / Lars Alberth / Larissa Schindler (Hg.), Materialitäten der Kindheit. Körper - Dinge - Räume. Wiesbaden: Springer VS, 249-261.

Selle, Klaus (2011): „Particitainment“ oder: Beteiligen wir uns zu Tode? Wenn alle das Beste wollen und Bürgerbeteiligung dennoch zum Problem wird. In: Planung neu denken online 3/2011. http://publications.rwth-aachen.de/record/140376/files/2011_selle_ particitainment.pdf (letzter Zugriff am 5.10.2021).

Shelton, Taylor / Lodato, Thomas (2019): Actually existing smart citizens. In: City 23/1, $35-52$.

Shelton, Taylor / Zook, Matthew / Wiig, Alan (2015): The „actually existing smart city“. In: Cambridge Journal of Regions, Economy and Society 8/1, 13-25.

Singer, Katrin (2019): Confluencing Worlds. Skizzen zur Kolonialität von Kindheit, Natur und Forschung im Callejón de Huaylas, Peru. Hamburg.

Skelton, Tracey (2010): Taking young people as political actors seriously. Opening the borders of political geography. In: Area 42/2, 145-151.

Skelton, Tracey / Aitken, Stuart C. (Hg.) (2019): Establishing geographies of children and young people. Singapore: Springer.

Smarter Together - gemeinsam g'scheiter (2020): Über Smarter Together. https:// www. smartertogether.at/ ueber-smarter-together/ (letzter Zugriff am 26.11.2020).

Söderström, Ola / Paasche, Till / Klauser, Francisco (2014): Smart cities as corporate storytelling. In: City 18/3, 307-320.

Späth, Philipp / Knieling, Jörg (2018): Endlich Smart-City-Leuchtturm. Auswirkungen des EU-Projektes mySMARTLife auf die Planungspraxis in Hamburg. In: Sybille Bauriedl / Anke Strüver (Hg.), Smart City. Kritische Perspektiven auf die Digitalisierung in Städten. transcript, 345-356.

Stadt Wien (2020): Wien stärkt Kinder- und J ugendmitbestimmung. https:// www.wien. gv.at/menschen-gesellschaft/kinder-und-jugendstrategie.html (letzter Zugriff am 11.1.2021).

Stadt Wien, J ugendabteilung (2020): Die Wiener Kinder- und J ugendstrategie. Wien.

Stadt Wien, Stadtentwicklung und Stadtplanung (2014): Smart City Wien Rahmenstrategie.

Taylor, Affrica (2011): Reconceptualizing the „nature“ of childhood. In: Childhood 18/4, 420-433.

The Smart Cities Information System (2020): Smart Cities and Communities Lighthouse projects. https:// smart-cities-marketplace.ec.europa.eu/ (letzter Zugriff am 31.8.2021).

Tonucci, Francesco / Rissotto, Antonella (2001): Why do we need children's participation? The importance of children's participation in changing the city. In: J ournal of Community and Applied Social Psychology 11/ 6, 407-419.

UNICEF (2020): Child Friendly Cities Initiative. https:// childfriendlycities.org/ what-isthe-child-friendly-cities-initiative/ (letzter Zugriff am 25.11.2020).

Valentine, Gill / Holloway, Sarah L. (Hg.) (2000): Children's geographies. Playing, living, learning. London/New York: Routledge.

van der Graaf, Shenja (2020): The right to the city in the platform age. Child-friendly city and smart city premises in contention. In: Information 11/6, 1-16.

Vanolo, Alberto (2014): Smartmentality. The smart city as disciplinary strategy. In: Urban Studies Journal Limited 51/5, 883-898.

Vanolo, Alberto (2018): Cities and the politics of gamification. In: Cities 74, 320-326.

Zeiher, Helga (2009): Ambivalenzen und Widersprüche der Institutionalisierung von Kindheit. In: Michael-Sebastian Honig(Hg.), Ordnungen der Kindheit. Problemstellungen und Perspektiven der Kindheitsforschung. Weinheim/München: Juventa, 103-126.

Zeiher, Helga (2018): Kindheit und Stadträume - Wandel in den letzten Jahrzehnten. In: Informationen zur Raumentwicklung 2/2018, 28-39.

Zinnecker, Jürgen (1990): Vom Straßenkind zum verhäuslichten Kind. In: Imbke Behnken (Hg.), Stadtgesellschaft und Kindheit im Prozeß der Zivilisation. Konfigurationen städtischer Lebensweise zu Beginn des 20. Jahrhunderts. Wiesbaden: VS Verlag für Sozialwissenschaften, 142-162. 


\section{Dokumente, Interviews und Gruppendiskussionen}

\section{Arbeitsprogramme Horizon 2020}

AP1European Commission: Horizon 2020 Smart, Green and Integrated Transport: Horizon 2020 Work Programme 2014-2015. https:// ec.europa.eu/research/participants/ data/ ref/h2020/wp/2014_2015/main/h2020-wp1415-transport_en.pdf (letzter Zugriff am 31.8.2021).

AP2 European Commission: Horizon 2020 Smart, Green and Integrated Transport: Horizon 2020 Work Programme 2018-2020. https:// ec.europa.eu/research/participants/ data/ ref/h2020/wp/2018-2020/main/h2020-wp1820-transport_en.pdf (letzter Zugriff am 31.8.2021).

\section{Smart-City-Projektdarstellung des Community Research and Development Information Service}

COR1 CORDIS: RUGGEDISED. Fact Sheet. https://cordis.europa.eu/project/id/731198 (letzter Zugriff am 3.6.2020).

\section{Smart-City-Projektwebsites}

W1SHARING CITIES: Vision. www.sharingcities.eu/ (letzter Zugriff am 5•3.2020)

W2 SMARTEN CITY: Tartu. https:// smartencity.eu/ about/lighthouse-cities/ tartu-estonia/ (letzter Zugriff am 8.5.2020).

W3 MATCH UP: News. Good practices start from schools: MAtchUP kicks off its study tours in Valencia to educate critical citizens. http://www.matchup-project.eu/news/ good-practices-start-from-schools-matchup-kicks-off-its-study-tours-in-valencia-toeducate-critical-citizens/ (letzter Zugriff am 2.9.2020).

W4 SMARTER TOGETHER: Simmering: München.de (2019): Schnitzeljagd mal anders. https:/ / www.muenchen.de/ rathaus/ Stadtverwaltung/ Referat-fuer-Arbeit-undWirtschaft/Europa/Smart-Cities/News-Smarter-Together-M-nchen/Kreuz-und-Quer. html (letzter Zugriff am 2.3.2020).

W5 SHARING CITIES: Milan. www.sharingcities.eu/sharingcities/city-profiles/milan (letzter Zugriff am 5·3.2020).

W6 IRIS: The Iris Smart Cities Concept and Approach underpinning the project. https:// www.irissmartcities.eu/ content/ iris-smart-cities-concept-approach-underpinningproject (letzter Zugriff am 31.8.2021).

W7 IRIS: Context. https://www.irissmartcities.eu/content/context (letzter Zugriff am 31.8.2021).

\section{Newsletter (NL) Smart-City-Leuchtturmprojekte der EU}

NL1 SMARTER TOGETHER Simmering: Nr. 30, 11/2018.

NL2 SMARTER TOGETHER Simmering: Nr. 28, 09/2018.

NL3 SMARTER TOGETHER Simmering: Nr. 41, 09/2020.

NL4 SMARTER TOGETHER Simmering: Nr. 36, o8/2019.

NL5 SMARTER TOGETHER Simmering: Nr. 18, 11/ 2017.

NL6 TRIANGULUM: Nr. 6, 10/2017.

NL7 SMARTER TOGETHER Simmering: Nr. 25, 06/ 2018.

NL8 SMARTER TOGETHER Simmering: Nr. 12, 07/2017.

NL9 STARDUST: Nr. 2, 03/2019.

NL10 TRIANGULUM: Nr. 2, 04/ 2017.

NL11 SMARTER TOGETHER Simmering: Nr. 4, 11/ 2016.

NL12 SMARTEN CITY: Nr. 19, 04/2020.

NL13 SMARTER TOGETHER Simmering: Nr. 38, 10/2019. 
Teilnehmende Beobachtung

TB1 Auftaktveranstaltung Mobilitätsspiel „Street-Points“, Wien-Favoriten, 7.10.2019.

Interviews (IN), Gruppendiskussionen (GD), informelles Gespräch (IG)

IN1 Mitarbeiter_in des EU-Smart-City-Leuchtturmprojekts „Smarter Together“ Simmering, 13.9.2019.

IN2 Mitarbeiter_in desEU-Smart-City-Leuchtturmprojekts „SmarterTogether“ “Simmering, 10.9.2019.

IN3 Mitarbeiter_in der Planungsdirektion der Stadt Wien, 10.8.2020.

IN4 Mitarbeiter_in im Projekt „Smart City Wien“, Magistratsabteilung Stadtentwicklung und Stadtplanüng, 8.10.2019.

IN5 Mitarbeiter_in der Geschäftsgruppe Bildung, Integration, Jugend und Personal der Stadt Wien, 11.9.2019.

GD1 Fünf Teilnehmer_innen der offenen Kinder- und Jugendarbeit, 10/2019.

GD2 Zwei Teilnehmer_innen der offenen Kinder- und Jugendarbeit, 08/2020.

IG1 Informelles Gespräch mit Teilnehmer_innen der offenen Kinder- und Jugendarbeit, $08 / 2020$.

\section{Smart Childhoods.}

\section{When young people become the focus of urban governance}

The smart city vision comprises the hope of overcoming pressing ecological, economic and social urban crises, as well as the aspiration to transition to a resource-saving and livable city of the future. It is conspicuous that smart city initiatives specifically address children and young people. Drawing on approaches from governmentality and geographical childhood studies, we explore how the practically unchallenged ideal of a city shaped by innovation optimism changes notions of childhood and drives its social valorization. First weuse discourseanalysis to examine how EU smart city lighthouse projects invest in children's immediate environment and use it as a laboratory for smart urban development. Wethen discuss how smart city refurbishment influences the everyday lives of children and young people and how it adresses their needs. For this we draw on a case study of the smart city project Vienna Simmering. Our results show that young people, as smart citizens, are obliged to support urban development policy objectives and to implement the corresponding courses of action into their everyday lives. This results in new relationships of dependency that need to be critically examined. 
